\title{
Relationship Between Structure and Function of Neurons in the Rat Rostral Nucleus Tractus Solitarii
}

\author{
MICHAEL S. KING AND ROBERT M. BRADLEY \\ Department of Biologic and Materials Sciences, School of Dentistry (M.S.K., R.M.B.) and \\ Department of Physiology, School of Medicine (R.M.B.), University of Michigan, \\ Ann Arbor, Michigan 48109-1078
}

\begin{abstract}
To investigate the relationship between the structure and function of neurons in the rostral (gustatory) nucleus tractus solitarii (rNTS), we analyzed the morphological and biophysical properties of rNTS neurons by performing whole-cell recordings in a brain slice preparation. Overall, neurons $(n=58)$ had a mean somal diameter of $16 \mu \mathrm{m}$, an average dendritic length of $598 \mu \mathrm{m}$, an average dendritic thickness of $0.91 \mu \mathrm{m}$, and a spine density of 0.037 spines $/ \mu \mathrm{m}$. Neurons were separated into three groups (elongate, multipolar, and ovoid) on the basis of previously established morphological criteria. The highest percentage (49\%) of neurons were classified as ovoid, while $35 \%$ were multipolar and only $16 \%$ were elongate.

The most frequently observed firing pattern, in all three cell types, elicited by a $1,200 \mathrm{~ms}$, $100 \mathrm{pA}$ depolarizing current pulse was a regularly firing spike train. However, the intrinsic firing properties of the remaining neurons were different. Thirty-one percent of the ovoid neurons responded with a short burst of action potentials and $44 \%$ of the elongate neurons showed a delay in the onset of the spike train following a hyperpolarizing prepulse. Less than $16 \%$ of the multipolar neurons demonstrated either of these firing characteristics.

Therefore, rNTS neurons with similar morphology do not have unique biophysical properties. However, the data suggest that there may be subpopulations of the three morphological types, each of which displays a different firing pattern. Since the structure and function of the three morphological groups were not strictly correlated, these subpopulations may represent functional groups. 1994 Wiley-Liss, Ine.
\end{abstract}

Key words: biocytin, brain slice, taste, whole-cell recordings, neuron reconstruction

Chemosensory information from the oral cavity, pharynx, and larynx travels in the facial, glossopharyngeal and vagus nerves to the ipsilateral nucleus tractus solitarii (NTS), a longitudinal column of neurons extending from the first cervical segment of the spinal cord to the genu of the facial nerve (Torvik, 1956; Hamilton and Norgren, 1984; Bradley et al., 1985; Housley et al., 1987). The facial nerve projection terminates in the rostral pole of the NTS (rNTS), the glossopharyngeal nerve terminates caudal to the facial nerve, and the vagus nerve terminates caudal to the glossopharyngeal nerve. Chemosensory information carried by the facial and glossopharyngeal nerves is believed to be gustatory in nature while that conveyed by the vagus nerve appears to play a major role in upper airway protective reflexes (Bradley et al., 1983; Smith and Hanamori, 1991).

The gustatory input of the rNTS has been defined in some detail with neural tracing techniques. The chorda tympani projection terminates medial to the solitary tract in a region called the rostral central subdivision (Whitehead and Frank, 1983; Whitehead, 1988). This subdivision is also the site of termination of the lingual branch of the glossopharyngeal nerve and is therefore hypothesized to be the major gustatory recipient zone of the rNTS. The projection zones of these two taste nerves are not fully segregated since their distributions overlap within the rNTS (Hamilton and Norgren, 1984).

Analysis of Golgi-stained material of the gustatory zone of the rat and hamster rNTS has shown that it contains at least three neuron types (Davis and Jang, 1988; Lasiter and Kachele, 1988; Whitehead, 1988; King and Hill, 1993).

\footnotetext{
Accepted December 26, 1993

Michael S. King is now at Campus Box 8264, Biology Department, Stetson University, DeLand, FL 32720-3756.

Address reprint requests to Dr. Robert M. Bradley, 6228 School of Dentistry, 1011 N. University Ave., University of Michigan, Ann Arbor, MI 48109-1078
} 
Multipolar (stellate) neurons have a triangular or polygonalshaped soma and three to five primary dendrites; elongate (fusiform) neurons are characterized by an elongated soma and two thick primary dendrites that exit the cell body at opposite poles; and ovoid neurons have small somal diameters and two to four primary dendrites that are thin and sparsely branched. Based on neural tracing studies these different neuron types have been demonstrated to have different projection patterns. Multipolar and elongate neurons project rostrally to the pontine gustatory relay (Lasiter and Kachele, 1988; Whitehead, 1990). Multipolar neurons in the ventral part of rNTS project to the reticular formation and the motor nuclei of cranial nerves V, VII, IX, X, and XII (Norgren, 1978; Travers, 1988; Beckman and Whitehead, 1991). Thus, rostrally directed neurons are believed to be important in processing and relaying gustatory information while caudally connected neurons are presumably involved in the reflex control of salivation and ingestive behavior (Beckman and Whitehead, 1991). The ovoid neurons are believed to be interneurons because they are relatively small, and in labeling studies they do not project to the pontine taste relay, the caudal NTS, or the medullary reticular formation (Lasiter and Kachele, 1988; Davis, 1993).

Neurons of rNTS have also been separated into four different groups based on their repetitive firing properties (Bradley and Sweazey, 1992). However, the morphology of these neurons was not determined so that the biophysical properties of intrinsic and projection neurons is unknown. We therefore used whole-cell recordings in brain slices of rNTS combined with intracellular labeling to determine the intrinsic firing properties of morphologically identified neurons. This information is important in understanding the neural circuits involved in processing gustatory information at the first central relay in the taste pathway. Some of these results have been presented in preliminary form (King and Bradley, 1993a,b).

\section{MATERIALS AND METHODS Brain slice preparation}

Brain slices containing the rostral $1.6 \mathrm{~mm}$ of the NTS were obtained from 45 female Sprague-Dawley rats (Charles River) weighing 125 to 175 grams, as previously described (Bradley and Sweazey, 1990,1992). Rats anesthetized with sodium pentobarbital $(50 \mathrm{mg} / \mathrm{kg})$ were decapitated and the brainstem quickly removed and cooled in physiological saline for 2 minutes. The physiological saline contained (in mM) NaCl 124, $\mathrm{KCl} 5, \mathrm{CaCl}_{2} 2.5, \mathrm{MgSO}_{4} 1.3, \mathrm{NaHCO}_{3} 26$, $\mathrm{KH}_{2} \mathrm{PO}_{4} 1.25$, and dextrose 25 . The solution was gassed with $95 \% \mathrm{O}_{2}$ and $5 \% \mathrm{CO}_{2}$ to maintain the $\mathrm{pH}$ at 7.4. After cooling, the brainstem was secured onto the stage of a Vibratome with cyanoacrylate glue and cut into $400 \mu \mathrm{m}$ thick coronal or sagittal slices or $300 \mu \mathrm{m}$ thick horizontal slices. Before recording, the slices were incubated for at least 1 hour in physiological saline at room temperature. During recordings, slices were secured in a chamber with nylon mesh and continuously superfused with oxygenated saline flowing at 1.5 to $2 \mathrm{ml} / \mathrm{min}$. By a system of valves, physiological saline containing $1 \mathrm{M}$ substance $\mathrm{P}(\mathrm{SP}), 1 \mathrm{mM}$ $\gamma$-aminobutyric acid (GABA), or $100 \mu \mathrm{M} N$-methyl-Daspartate (NMDA; all obtained from Sigma), was superfused over the slice for 30 seconds, followed by superfusion with physiological saline. Throughout the experiments the temperature was maintained between 31 and $33^{\circ} \mathrm{C}$.

\section{Whole-cell recordings and biophysical measurements}

Unpolished patch electrodes were pulled in two stages on a Narishige PP83 electrode puller from $1.5 \mathrm{~mm}$-OD borosilicate filament glass (WPI, TW 150F-4). The electrodes had tip resistances of 5-8 M 2 when filled with a solution containing (in mM) K gluconate 130, Hepes 10, EGTA 10, $\mathrm{MgCl}_{2} 1, \mathrm{CaCl}_{2} 1, \mathrm{ATP} 2$, and $1 \%$ biocytin. The $\mathrm{pH}$ was adjusted to 7.4 with $\mathrm{KOH}$. Whole-cell recordings were made using the technique of Blanton et al. (1989), as previously described in detail (Bradley and Sweazey, 1992). Once a giga- $\Omega$ seal was formed and the patch ruptured, current stimulation protocols were performed and voltage data were acquired using the pCLAMP program (Axon Instruments). Current was injected into neurons using the bridge circuit of an Axoclamp 1A amplifier in current clamp mode. The indifferent electrode was $\mathrm{Ag}-\mathrm{AgCl}$ wire connected to the extracellular solution via an agar bridge.

Electrophysiological data were analyzed using the Clampfit program (Axon Instruments). Biophysical properties, such as input resistance, action potential amplitude, width, rise time and latency, and membrane time constants were determined by delivering a series of $100 \mathrm{~ms}$ hyperpolarizing and depolarizing current pulses $(-100$ to $75 \mathrm{pA})$. Since recordings were made with a potassium gluconate solution in the recording pipette, a junctional potential of $10 \mathrm{mV}$ was subtracted from the membrane potential values (Staley and Mody, 1992). To be included in the electrophysiological analysis neurons had to have a resting membrane potential more negative than $-40 \mathrm{mV}$, an action potential overshoot, and an input resistance greater than $100 \mathrm{M} \Omega$.

Neurons were separated into four groups on the basis of their repetitive firing pattern induced by a $1,200 \mathrm{~ms}, 100 \mathrm{pA}$ depolarizing current pulse, which was given alone or preceded by a $-200 \mathrm{pA}$ hyperpolarizing pulse of increasing duration $(50,100$, and $150 \mathrm{~ms})$. The four biophysical groups have been described previously in detail (Bradley and Sweazey, 1992). Most rNTS neurons fire a repetitive spike train throughout the long-lasting $(1,200 \mathrm{~ms})$ depolarizing current pulse (groups I, II, and III). In a smaller population of neurons (group IV), the discharge pattern consists of a short burst of action potentials which occurs at the onset of the depolarization. The firing patterns of group I, II, and III neurons are distinguished by hyperpolarizing the neurons prior to delivering the long depolarizing current pulse. In group I neurons, the repetitive discharge pattern initiated by membrane depolarization is changed to an irregular spike train by prior hyperpolarization. In group II neurons, hyperpolarization either delays the occurrence of the first action potential or increases the length of the first interspike interval in the action potential train produced by membrane depolarization. In group III neurons, hyperpolarization has little effect on the repetitive discharge pattern.

The effects of SP, GABA, and NMDA on neural activity of several neurons in each group was also examined (see legend to Fig. 14 for $n$ values). In these experiments we determined that a neuron responded to a neurotransmitter if a change in membrane potential $(>1 \mathrm{mV})$ occurred with the application of the neurotransmitter.

\section{Intracellular labeling with biocytin}

To determine the morphology of the biophysically characterized neurons, $1 \%$ biocytin (Sigma; Horikawa and Armstrong, 1988) was diluted in the pipette-filling solution and 
placed in the tip of the recording pipette. The neurons were filled with biocytin by diffusion. The biocytin did not affect the biophysical properties of the neurons, as compared with our previous studies in brain slices without biocytin (Bradley and Sweazey, 1992; King et al., 1993; Wang and Bradley, 1993). Following the experiments, the seal was broken by delivering several large hyperpolarizing current pulses $(-4$ to $-6 \mathrm{nA}, 50 \mathrm{~ms}$; Berger et al., 1992), and the slices were removed from the recording chamber, placed on a piece of filter paper and fixed in $10 \%$ neutral-buffered formalin for at least 24 hours.

After fixation, slices were rinsed in $0.1 \mathrm{M}$ phosphate buffer for 30 minutes, embedded in agar (4\% in distilled $\mathrm{H}_{2} \mathrm{O}$ ), and cut into $100 \mu \mathrm{m}$ thick sections on a Vibratome. The sections were incubated for 2 hours in avidinhorseradish peroxidase (avidin-HRP; Sigma) at room temperature in the dark. The avidin-HRP was diluted 1:200 in phosphate-buffered saline containing $0.3 \%$ Triton-X (Sigma). The sections then were rinsed for 30 minutes and reacted with $0.025 \%$ diaminobenzidine and $0.01 \% \mathrm{H}_{2} \mathrm{O}_{2}$ for $6-10$ minutes. After rinsing, the sections were mounted on gelatin-coated slides, dried overnight, and coverslipped using Krystalon mounting medium (Harleco Co.). To minimize tissue shrinkage, the sections were not dehydrated in alcohols nor cleared in xylenes. Of 95 neurons that were recorded, $58(61 \%)$ were satisfactorily filled to allow characterization and reconstruction.

\section{Reconstruction of neurons}

Fifty-eight neurons were reconstructed in three dimensions using the computerized Eutectic Neuron Tracing System (Eutectic Electronics, Inc.). During reconstruction, neurons were visualized with a Zeiss $100 \times$ objective under oil (final magnification of $1,000 \times$ ), enabling the identification of detailed morphological features, such as spines. Neurons were reconstructed by manually moving a cursor along the length of a neuron's dendrites and having the computer assign an $\mathrm{X}, \mathrm{Y}$, and $\mathrm{Z}$ value to note position as well as a diameter to a series of points along the dendrites. The position of branch points, spine bases, and endings was also recorded. All of the neurons had processes which extended into at least two sections, and therefore, the reconstruction also involved determining how the more distal processes connected to the neural structures in the preceding section. Camera lucida drawings of the neural structures in each section were overlapped to facilitate the determination of how the processes were connected. Following reconstruction, the Z-axis was expanded so that the extent of the neuron in the Z-plane corresponded to the thickness of the sections in which neural structures were present. The position of each neuron within the NTS was recorded by tracing the outline of the nucleus at low power (final magnification of $63 \times$ ).

\section{Morphometric analysis}

Using the Eutectic System, for each neuron we measured somal diameter, area and form factor, dendritic length, thickness and volume, primary segment length, spine density, orientation angle, and area and volume of the region of influence of a neuron's dendritic trees. Somal form factor is a measure of the roundness of the cell body, where 1 indicates a perfect circle and 0 indicates a line. This parameter is calculated as $(4 \times$ area) divided by the perimeter. ${ }^{2}$ Total dendritic length is the sum of the lengths of all of the dendrites of an individual neuron. Average dendritic length is the total dendritic length divided by the number of primary dendrites. The primary segment length is the distance from the edge of the soma to the first branch point of each primary dendrite. This measurement was given as an average for all the primary dendrites of an individual neuron. As an indication of how far a neuron's dendrites extended from its cell body, we calculated the shortest (or airline) distance between the soma and each of the dendritic endings. This measurement is called a tip distance, and for each neuron we noted the minimum, maximum, and mean tip distances.

To determine the orientation of each neuron within the rNTS, we measured the orientation angle in which a neuron's dendritic field was positioned. This measurement assigns an axis, in the $x-y$ plane, to the dendritic field of a neuron, averaging the position of each of the neuron's dendrites. The orientation angle ranges from 0 to $179^{\circ}$ and indicates the average direction of the dendritic field of a neuron, where $0^{\circ}$ indicates that the axis is oriented mediallaterally. An orientation angle of $90^{\circ}$ indicates that the axis is dorsal-ventral in coronal slices and rostral-caudal in horizontal slices. As another indication of the orientation of a neuron's dendrites within the rNTS, we determined the total length of dendrite for each neuron which extended in the medial-lateral, rostral-caudal, and dorsal-lateral directions. Finally, we devised a measurement to indicate the position from which a neuron's primary dendrites exited the soma. This parameter was calculated as the distance between the dendritic tree origins divided by the maximum soma diameter. In a prototypical elongate neuron, where two primary dendrites exit opposite poles of the cell body, this measurement is 1 . In neurons with more than two dendrites, this number is $<1$.

Neurons were assigned to one of three morphological groups (elongate, multipolar, and ovoid) by visual inspection based on previously established criteria in the rNTS (Lasiter and Kachele, 1988; Whitehead, 1988; King and Hill, 1993). Specifically, the criteria used were somal shape, number of dendrites, position from which the dendrites exit the soma, and dendritic thickness and branching. Elongate neurons had spherical to fusiform somata with two primary dendrites exiting opposite poles of the cell body. Multipolar neurons had pyramidal somata with three to five primary dendrites. Ovoid neurons had spherical to ovoid somata and two to five thin and relatively unbranched primary dendrites.

\section{Statistical analysis}

All statistical analysis was conducted using the SYSTAT program. Morphological properties of different neuron groups were compared by performing one-way analysis of variance with Tukey post-hoc tests. Correlations were determined using Bonferroni probabilities and the function was defined by the least-squares method, the line was fit by linear regression, and the statistical significance was determined by analysis of variance. A chi-square analysis was used to determine whether the relative numbers of elongate, multipolar, and ovoid neurons demonstrating the firing characteristics of the four biophysical groups of neurons were significantly different. In an attempt to identify natural groupings in the data, a cluster analysis was performed using the kmeans method and Euclidean distances. Data are presented as means \pm sem and statistical significance is reached when $P \leq .05$. 
A.

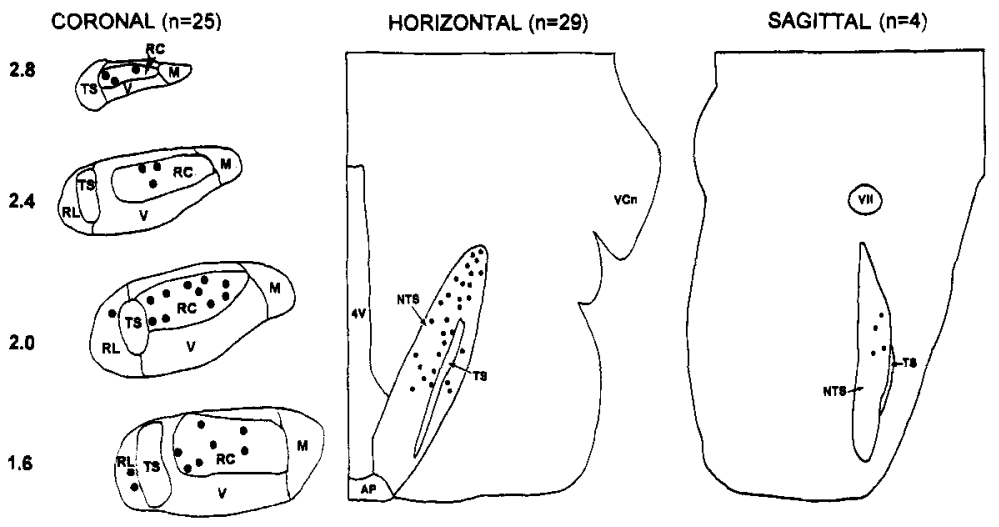

B.
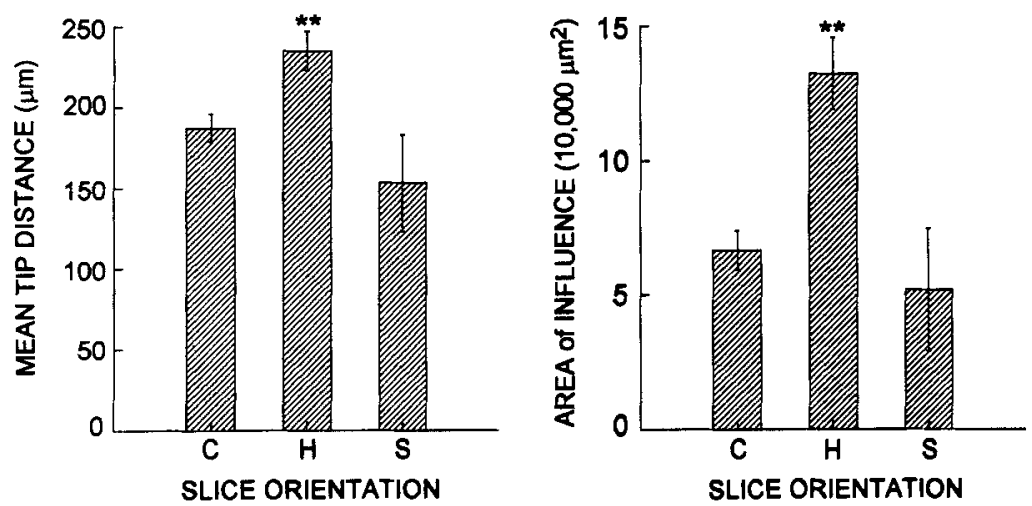

Fig. 1. A: Schematic diagrams of the locations of the 58 neurons included in this study. For each slice orientation a dot represents a single neuron. For the coronal slices the approximate boundaries of the cytoarchitectonic subdivisions of the rostral nucleus tractus solitarii (rNTS) are indicated based on Whitehead's (1988) description in the hamster. The numbers on the left of the coronal sections indicate millimeters rostral to the obex. AP, area postrema; $M$, medial subdivision; NTS, nucleus tractus solitarii; TS, tractus solitarius; RC, rostra

\section{RESULTS \\ Location of labeled neurons}

Only neurons that were located rostral to the point where the medial border of the NTS separates from the lateral edge of the fourth ventricle were included in this study. This region is innervated by gustatory nerves (Hamilton and Norgren, 1984) and we refer to it as the rNTS. The approximate location of the 58 neurons included in this study is indicated in Figure 1A. The majority of neurons (69\%), were located 1.6 to $2.0 \mathrm{~mm}$ rostral to obex. In the coronal slices most neurons were recorded from the rostral central subdivision, as defined in hamster (Whitehead, 1988) while the rest were located in the rostral lateral subdivision.

\section{Morphological properties of all labeled neurons}

Before separating neurons into morphological groups by visual inspection, we analyzed the morphological properties of all labeled neurons. This analysis provided a general description of the structure of rN'TS neurons and was used in an attempt to separate the neurons into groups on the basis of defined morphological parameters.

central subdivision; $\mathrm{RL}$, rostral lateral subdivision; $\mathrm{V}$, ventral subdivision; VCn, ventral cochlear nucleus; $4 \mathrm{~V}$, fourth ventricle; VII, genu of the VII nerve. B: Comparison of the mean tip distance and the area of influence of the dendritic trees of neurons in coronal (C), horizontal $(\mathrm{H})$, and sagittal (S) slices. Stars indicate that the values for neurons in horizontal slices were statistically greater than those for neurons in both coronal and sagittal slices.

Biocytin-filled neurons in the rNTS had a mean somal diameter $( \pm \mathrm{sem})$ of $16.1 \pm 4.0 \mu \mathrm{m}$, total dendritic length of $1,920 \pm 126 \mu \mathrm{m}$, average dendritic length of $598 \pm 36 \mu \mathrm{m}$, thickness of $0.91 \pm 0.04 \mu \mathrm{m}$, and a spine density of $0.037 \pm$ 0.003 spines $/ \mu \mathrm{m}$. On average, they had $3.4 \pm 0.1$ primary dendrites each of which branched an average of $2.4 \pm 0.2$ times. Most neurons (78\%) had dendrites with frequent swellings along their length giving them a beaded appearance. This was most often observed in the thinner processes. Many of the fiber swellings (20\%) had spines on them.

In general, morphological properties were not related to the orientation of the slice. However, although not statistically significant $(P=.07)$, neurons observed in horizontal slices tended to have longer dendrites $(684 \pm 69 \mu \mathrm{m})$ than neurons in coronal slices $(524 \pm 38 \mu \mathrm{m})$. This trend is reinforced by the fact that the mean tip distance and the area of influence of neurons in horizontal slices were significantly larger than in neurons in coronal or sagittal slices (Fig. 1B). This may result from the severing of rostral-caudal extending dendrites when preparing the 400 $\mu \mathrm{m}$ thick coronal slices indicating a predominate orientation of rNTS neurons in the horizontal plane (see the section on orientation below). 
A. ROSTRAL-CAUDAL POSITION
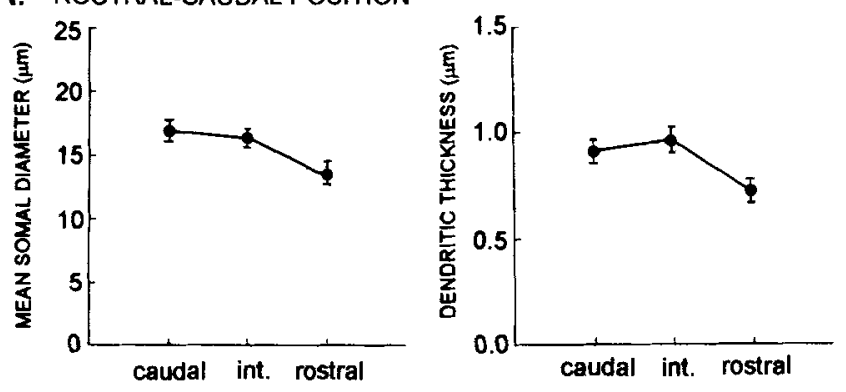

B. MEDIAL-LATERAL POSITION
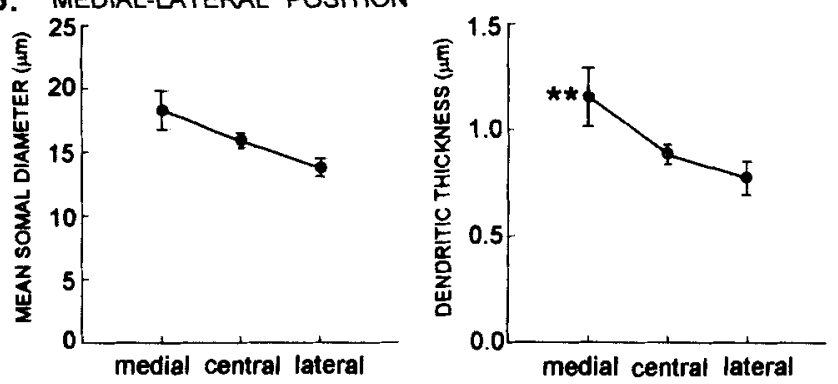

Fig. 2. Graphs of the mean somal diameter, dendritic thickness, volume of influence of the dendritic trees, and spine density versus neuron position within the rNTS. A: The rostral-caudal dimension of the rNTS was split into three regions: rostral (the rostral-most $400 \mu \mathrm{m}$; $\mathrm{n}=9$ ), intermediate (int; the middle $800 \mu \mathrm{m} ; \mathrm{n}=31$ ), and caudal (the caudal-most $400 \mu \mathrm{m} ; \mathrm{n}=18$ ). One asterisk indicates that the spine density of the more rostral neurons was significantly greater than that

A neuron's location within the rNTS was related to several morphological features (Fig. 2). Although most differences did not reach statistical significance they may be biologically significant. More rostral and lateral neurons tended to have smaller somal diameters, thinner dendrites, smaller volumes of influence, and more spines than neurons located more medially and caudally within the rNTS. For example, the most rostral neurons had the smallest minimum soma diameter and the greatest spine density (Fig. 2A; $P<.05$ ), and the most lateral neurons had the smallest maximum somal diameter, thinnest dendrites (Fig. 2B), and smallest dendritic volume $(P<.05)$.

We also examined the relationship among morphometric parameters by performing correlation analysis. Several correlations were determined, usually between related measurements such as dendritic length and area of influence. Figure 3 illustrates the relationship between somal area and dendritic thickness and volume. Neurons with larger somal areas had thicker dendrites $\left(\mathrm{r}^{2}=.531 ; P<.001\right)$ and a greater dendritic volume $\left(\mathrm{r}^{2}=.643 ; P<.001\right)$.

\section{Separation of neurons into morphological groups}

To classify neuron types within the rNTS we first attempted to identify groupings within the data by examining the distributions of morphological properties. All measurements displayed a unimodal distribution, as shown in Figure 4 for primary dendrite number, average dendritic length, and somal area. When examining the distribution of some morphometric parameters, occasionally a few outliers were identified (as for somal area, Fig. 4C), but this group
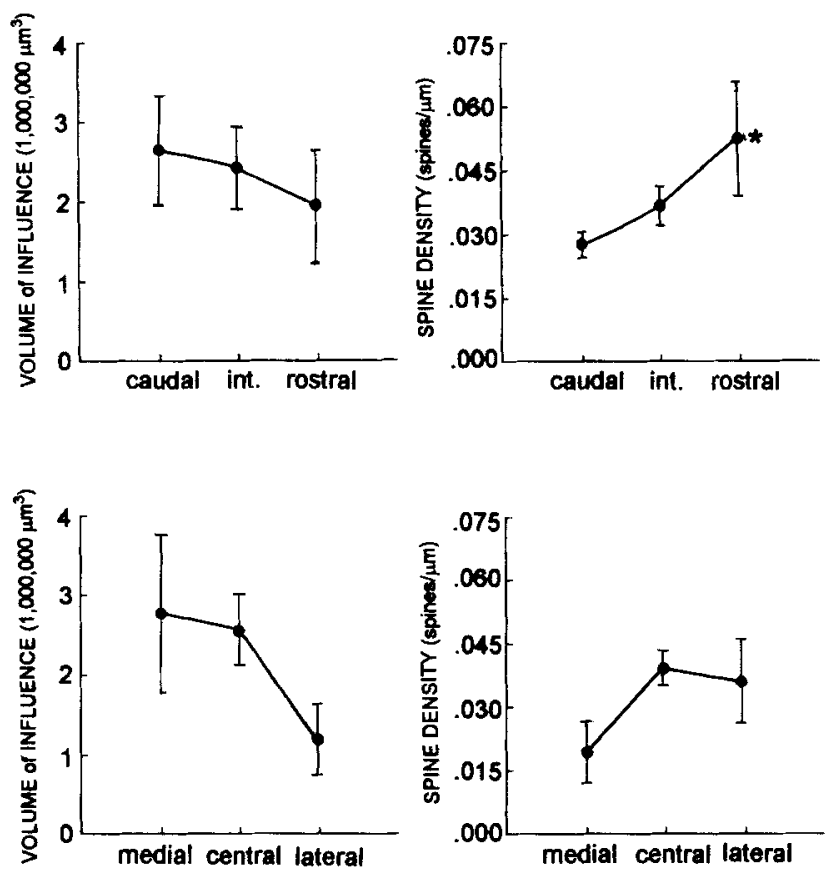

of the most caudal neurons. B: The medial-lateral dimension of the rNTS was split into three regions: lateral (lateral to the solitary tract $\mathrm{n}=7$ ), central (within and just medial to the solitary tract; $n=43$ ), and medial $(n=8)$. Two asterisks indicate that the dendritic thickness of the most medial neurons was significantly greater than that of the neurons located within the central and lateral rNTS.

never consisted of more than three or four neurons and did not produce a multimodal distribution. A cluster analysis also failed to identify distinct groupings in the morphometric data.

Therefore, to categorize neuron types within the rNTS we used previously established criteria where neurons are classified as elongate, multipolar, or ovoid by visual inspection of morphological features (Lasiter and Kachele, 1988; Whitehead, 1988; King and Hill, 1993). Examples of elongate, multipolar, and ovoid neurons are illustrated in Figure 5 and those neurons are reconstructed in Figure 6. Although the general morphology was consistent within groups, the size and shape of the neurons in the multipolar and elongate groups varied greatly. More specifically, although the somal shape of elongate neurons varied from spherical to fusiform, all elongate neurons had two primary dendrites that originated from opposite poles of the cell body. This is reflected in a dendritic tree origin distance/ maximum soma diameter ratio close to 1 (Table 1). Multipolar neurons were distinguished by their triangular or polygonal somata with three to five primary dendrites originating almost equidistantly from the cell body. Ovoid neurons had spherical to ovoid somata with two to five thin primary dendrites. Each neuron type was located throughout the rNTS and was not restricted to a particular rostral-caudal or medial-lateral location (Fig. 7).

\section{Morphological properties of elongate, multipolar, and ovoid neurons}

Several morphological properties were significantly different among the three groups (Fig. 8; Table 1). On average, 
A.

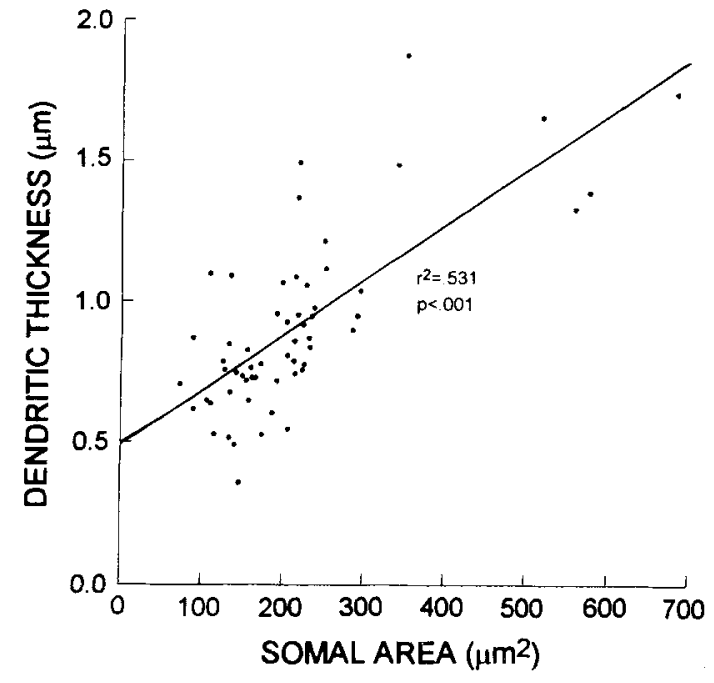

B.

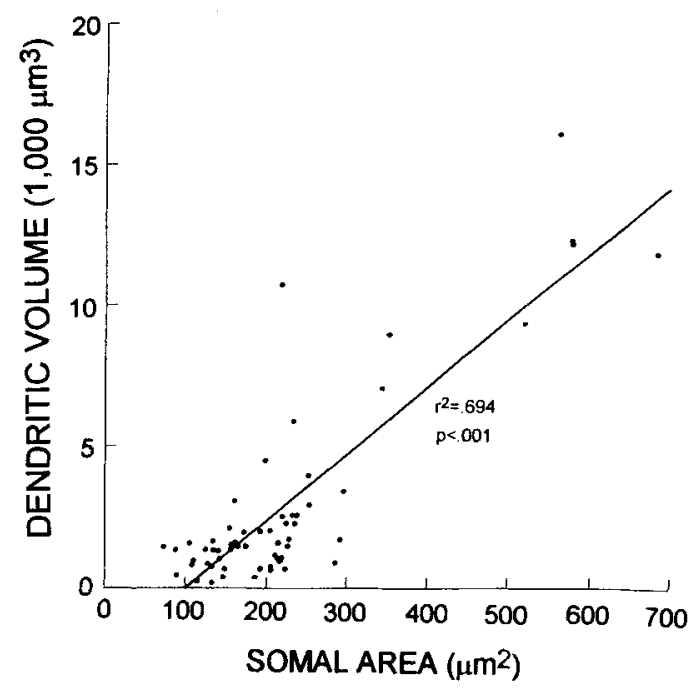

Fig. 3. Correlations between somal area and (A) dendritic thickness and (B) volume. Data were fitted by linear regression.

A.

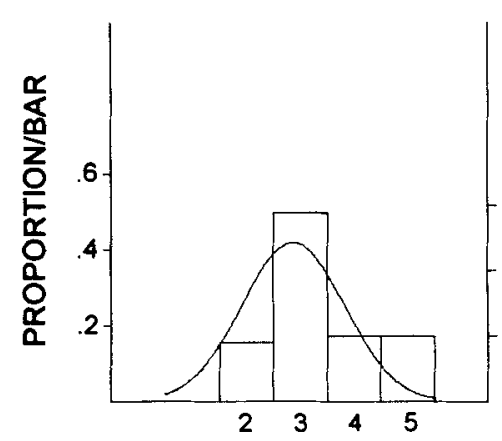

NUMBER of PRIMARY DENDRITES
B.

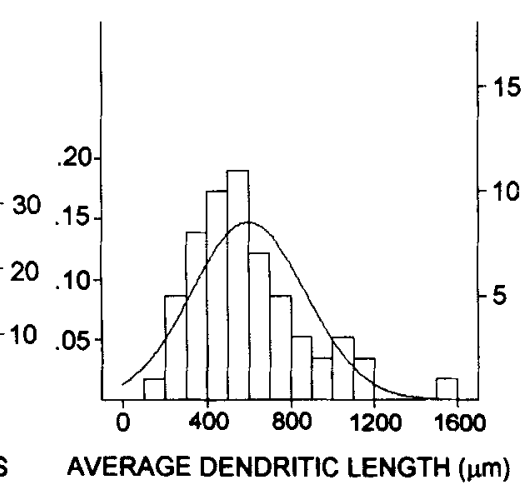

C.

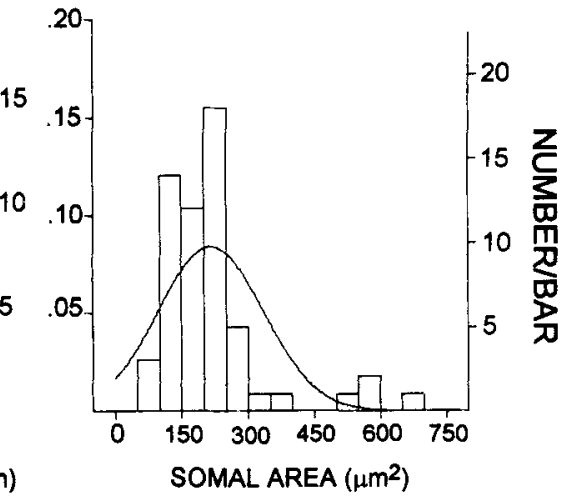

Fig. 4. Distributions of the (A) number of primary dendrites, (B) average dendritic lengths, and (C) somal areas for all 58 neurons. The proportion of the population represented by each bar is shown on the left ordinate and the total number of neurons represented by each bar is shown on the right. A normal curve is superimposed over the histograms for comparison.

elongate neurons had the most linear cell bodies with the greatest maximum somal diameters; the fewest, longest, and thickest primary dendrites which exited the soma at opposite ends; the shortest primary segment lengths; dendrites which terminated farthest from the soma; the most linear dendritic fields; and the fewest spines. Multipolar neurons had the most primary dendrites, the largest dendritic fields, the greatest dendritic volumes, and, the greatest spine density. Ovoid neurons had the smallest and most spherical cell bodies; the shortest, thinnest, and least branched dendrites; the smallest dendritic volumes; and the smallest volumes of influence. In addition, the distribution of spines along the dendrites of the three types of neurons was not the same (Fig. 9). While the spine density of ovoid and elongate neurons peaked between the second and fourth branch order, the higher branch orders in multipolar neurons had the greatest spine density. This increase in spine density in multipolar neurons was due to both an increase in the number of spines and shorter dendritic lengths of the higher branch order segments.

\section{Neuronal orientation}

By visual inspection of neurons in coronal and horizontal slices as well as three-dimensional rotational analysis of reconstructed neurons using the Eutectic System, we determined that rNTS neurons were preferentially oriented in the horizontal plane. Measurement of dendritic lengths revealed that 23 neurons had dendrites which extended predominantly in the rostral-caudal plane, 23 neurons had longer lengths of dendrite in the medial-lateral plane, and 12 neurons had dendrites extending predominately in the dorsal-ventral plane. Neurons predominantly oriented in the rostral-caudal direction had longer dendrites $(703 \pm 68$ $\mu \mathrm{m})$ than neurons with a medial-lateral $(557 \pm 46 \mu \mathrm{m}$; $P=.15)$ or dorsal-ventral $(474 \pm 53 \mu \mathrm{m}, P<.05)$ orientation. The dendrites of most neurons labeled in this study (93\%) remained within the rNTS, even when the soma was very close to a border of the rNTS. The four neurons with dendrites extending beyond the rNTS were in coronal slices with dendrites that either exited ventrolaterally into the 

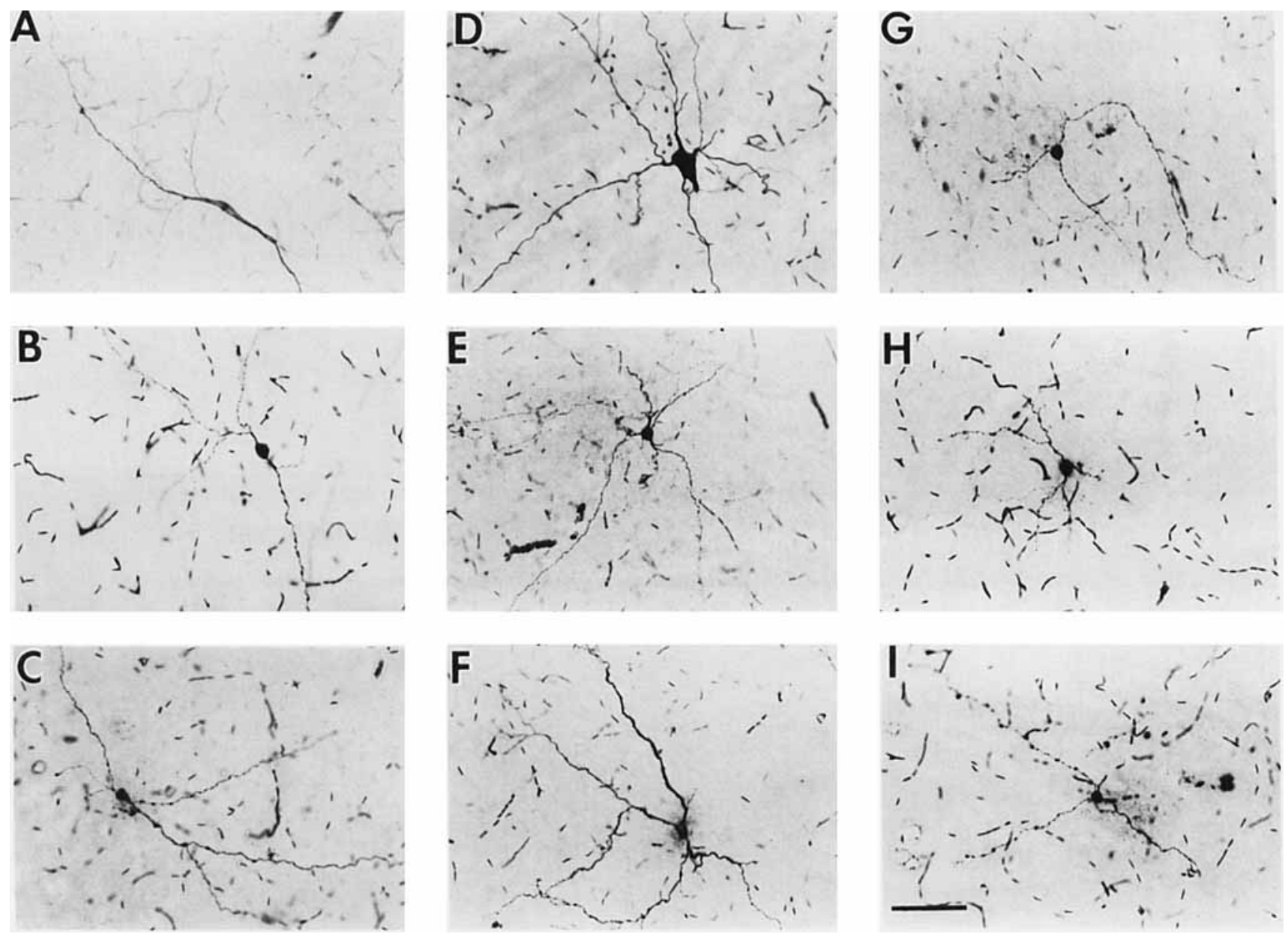

Fig. 5. Photomicrographs of three (A-C) elongate, (D-F) multipolar, and (G-I) ovoid neurons. All of the neurons except the one shown in $\mathrm{H}$ were in horizontal slices, where rostral is up and medial is to the right. The cell shown in $\mathrm{H}$ is in a coronal slice where up is dorsal and right is medial. Scale bar $=100 \mu \mathrm{m}$ and applies to $\mathrm{A}-\mathrm{I}$.

reticular formation ( $\mathrm{n}=3$ ) or dorsomedially into the medial vestibular nucleus ( $n=1$ ). Axons were identified as the thinnest processes exiting the soma or a primary dendrite. Axons in 30 neurons extended rostrally $(n=8)$, medially $(n=8)$, laterally $(n=6)$, caudally $(n=4)$, ventrally $(n=3)$, and dorsally $(n=1)$. The three cell types did not have a preferential axonal orientation. Most axons could only be followed a short distance and only one was observed leaving the rNTS.

The orientation angle for each neuron is shown in the scatter plots of Figure 10. In coronal slices, the dendrites of elongate and ovoid neurons had a predominantly dorsalventral orientation (angle near $90^{\circ}$ ), whereas those of multipolar neurons were oriented $45^{\circ}$ from the mediallateral direction (Fig. 10A). However, the large overlap in the scatter plots for each type of neuron indicates that this difference in the mean orientation angles was not significant. In horizontal slices, neurons of each group were oriented at an average angle of $145^{\circ}$ from the medial axis which is approximately parallel to the solitary tract (Fig. 10B). From the scatter plots in Figure 10B, it is apparent that only three neurons were not oriented parallel to the solitary tract.

\section{Biophysical properties of elongate, multipolar, and ovoid neurons}

Neurons included in this study had resting membrane potentials ranging from -40 to $-77 \mathrm{mV}$ with a mean of $-57 \pm 2 \mathrm{mV}$. Action potential amplitudes were between 48 and $90 \mathrm{mV}$ (mean $=71 \pm 2 \mathrm{mV}$ ) with a mean duration measured at half amplitude of $1.9 \pm 0.2 \mathrm{~ms}$. Input resistance ranged from 122 to $1,528 \mathrm{M} \Omega$ and averaged $494 \pm 44$ $M \Omega$. Neurons with the repetitive firing characteristics of group I $(n=6)$, group II $(n=8)$, group III $(n=20)$, and group IV $(\mathrm{n}=10)$ were recorded.

The repetitive firing properties of an elongate, multipolar, and ovoid neuron are shown in Figures 11-13, respectively. Each figure includes a reconstruction of the neuron (A), an illustration of its location within the rNTS (B), and a dendrogram, schematically illustrating the dendritic trees of the neuron (C). Cell types can usually be identified from their dendrogram because elongate neurons have few relatively elaborate dendrites, multipolar neurons have the most dendrites which are moderately branched, and ovoid neurons have the fewest dendrites which are relatively unbranched. The electrophysiological responses of the neu- 
A.

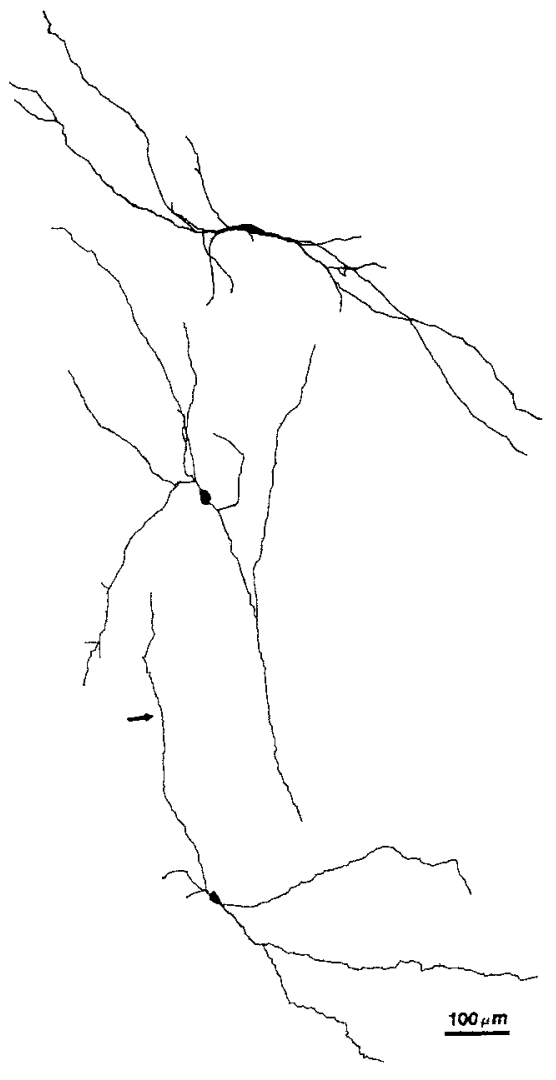

B.

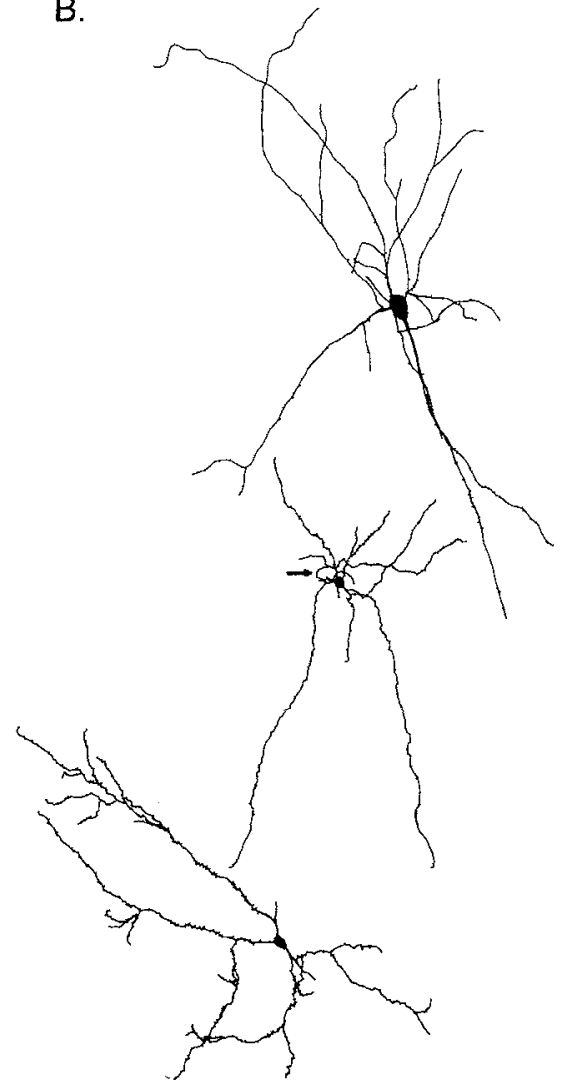

C.
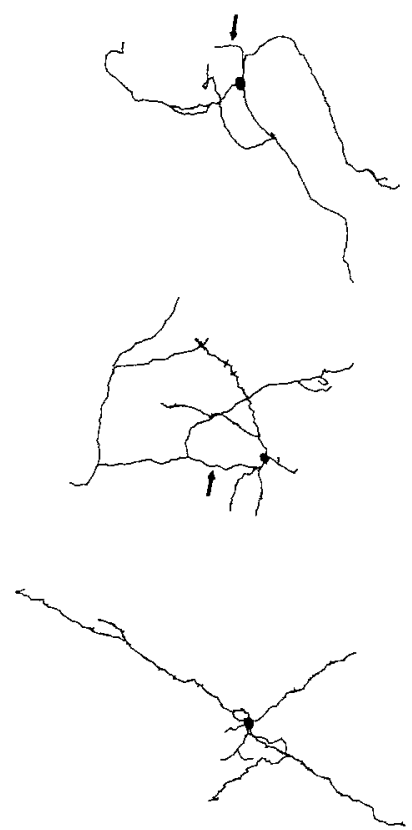

Fig. 6. Reconstructions of the (A) elongate, (B) multipolar, and (C) ovoid neurons shown in Figure 5. The orientations are as stated in the legend to Figure 5. Identified axons are indicated by the arrows.

A.

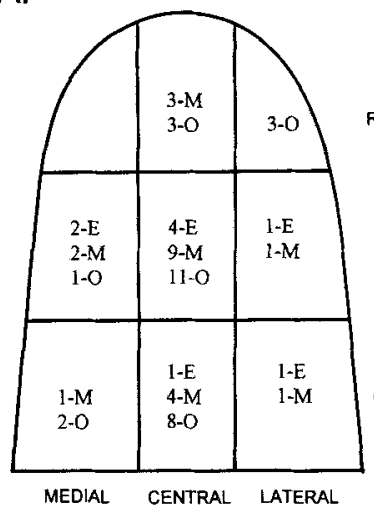

B.

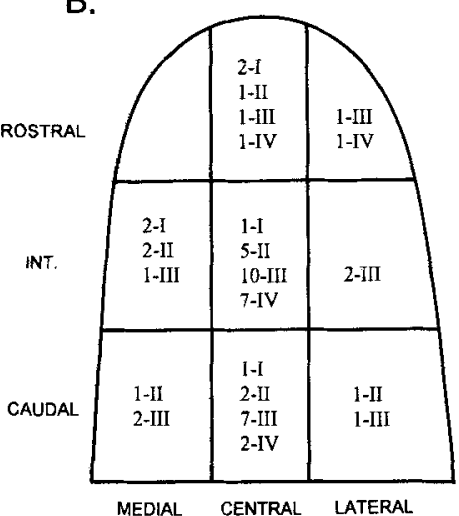

Fig. 7. Schematic representations of the rNTS split in the rostralcaudal dimension into caudal, intermediate (int.), and rostral regions and in the medial-lateral dimension into lateral, central, and medial regions (see legend to Fig. 2). A: The general location of neurons in each morphological group. The number and type of neuron ( $E$ is elongate, $M$ is multipolar, and $\mathrm{O}$ is ovoid) in each region is indicated. B: The general location of neurons in each biophysical group. The number of neurons in each group (I, II, III, or IV) is indicated in each region.

rons to depolarizing and hyperpolarizing current pulses are also illustrated in Figures 11-13 (D-F). These responses were used to determine the passive membrane properties and intrinsic firing characteristics of each neuron.

TABLE 1. Morphological Properties of Elongate, Multipolar, and Ovoid Neurons ${ }^{1}$

\begin{tabular}{|c|c|c|c|}
\hline $\mathrm{n}$ & $\begin{array}{l}\text { Elongate } \\
(n=9)\end{array}$ & $\begin{array}{l}\text { Multipolar } \\
(\mathrm{n}=21)\end{array}$ & $\begin{array}{c}\text { Ovoid } \\
(\mathrm{n}=28)\end{array}$ \\
\hline Primary dendrite number & $2.7 \pm 0.2^{\mathrm{m}}$ & $4.1 \pm 0.2^{\mathrm{e}, \mathrm{o}}$ & $3.1 \pm 0.1^{\mathrm{m}}$ \\
\hline Branches/dendrite & $2.9 \pm 0.6$ & $2.7 \pm 0.4$ & $2.0 \pm 0.2$ \\
\hline Total dendritic length $(\mu \mathrm{m})$ & $1,960 \pm 243$ & $2,629 \pm 228^{\circ}$ & $1,377 \pm 103^{\mathrm{m}}$ \\
\hline Primary segment length $(\mu \mathrm{m})$ & $58.2 \pm 23.2$ & $71.6 \pm 11.6$ & $79.7 \pm 9.8$ \\
\hline Minimum tip distance $(\mu \mathrm{m})$ & $70.5 \pm 13.3$ & $62.1 \pm 8.2$ & $51.3 \pm 4.8$ \\
\hline Maximum tip distance $(\mu \mathrm{m})$ & $441.9 \pm 49.1$ & $413.7 \pm 23.2$ & $355.8 \pm 29.1$ \\
\hline Mean tip distance $(\mu \mathrm{m})$ & $245.5 \pm 19.1^{\circ}$ & $227.6 \pm 11.8^{\circ}$ & $184.3 \pm 12.1^{e, m}$ \\
\hline Minimum somal diameter $(\mu \mathrm{m})$ & $13.3 \pm 1.4$ & $14.0 \pm 0.8$ & $13.2 \pm 0.4$ \\
\hline Maximum somal diameter $(\mu \mathrm{m})$ & $24.4 \pm 3.1^{\circ}$ & $21.5 \pm 1.6$ & $18.7 \pm 0.6^{\mathrm{e}}$ \\
\hline Mean somal diameter $(\mu m)$ & $16.7 \pm 1.5$ & $17.0 \pm 1.1$ & $15.2 \pm 0.4$ \\
\hline \multicolumn{4}{|l|}{ DTO distance/maximum somal } \\
\hline diamet & $0.90 \pm 0.04^{\mathrm{m}}$ & $0.74 \pm 0.02^{e}$ & $0.79 \pm 0.03$ \\
\hline Somal area $\left(\mu \mathrm{m}^{2}\right)$ & $232 \pm 43$ & $248 \pm 36$ & $186 \pm 10$ \\
\hline Dendritic volume $\left(\mu \mathrm{m}^{3}\right)$ & $3,189 \pm 997$ & $4,630 \pm 993^{\circ}$ & $1,222 \pm 151^{\mathrm{m}}$ \\
\hline
\end{tabular}

${ }^{1}$ Data are means \pm sem. See Materials and Methods for a description of the measurements. DTO, dendritic tree origin. Superscript letters indicate a significant difference from the elongate (e), multipolar (m) and/or ovoid (o) groups.

The passive membrane properties of the three morphological types of rNTS neurons were similar, except that the input resistance of the ovoid neurons was highest (Table 2). However, the frequency of repetitive firing patterns elicited by a $1,200 \mathrm{~ms}$ depolarizing current pulse, which was given alone or preceded by a hyperpolarizing pulse, was different among the morphological groups. Although no firing pattern was exclusive to all neurons in a morphological group, there were differences in the proportion of elongate, multipolar, and ovoid neurons in each of the biophysical groups $\left(\chi^{2}=102\right.$ for elongates, 43 for multipolars, and 24 for 

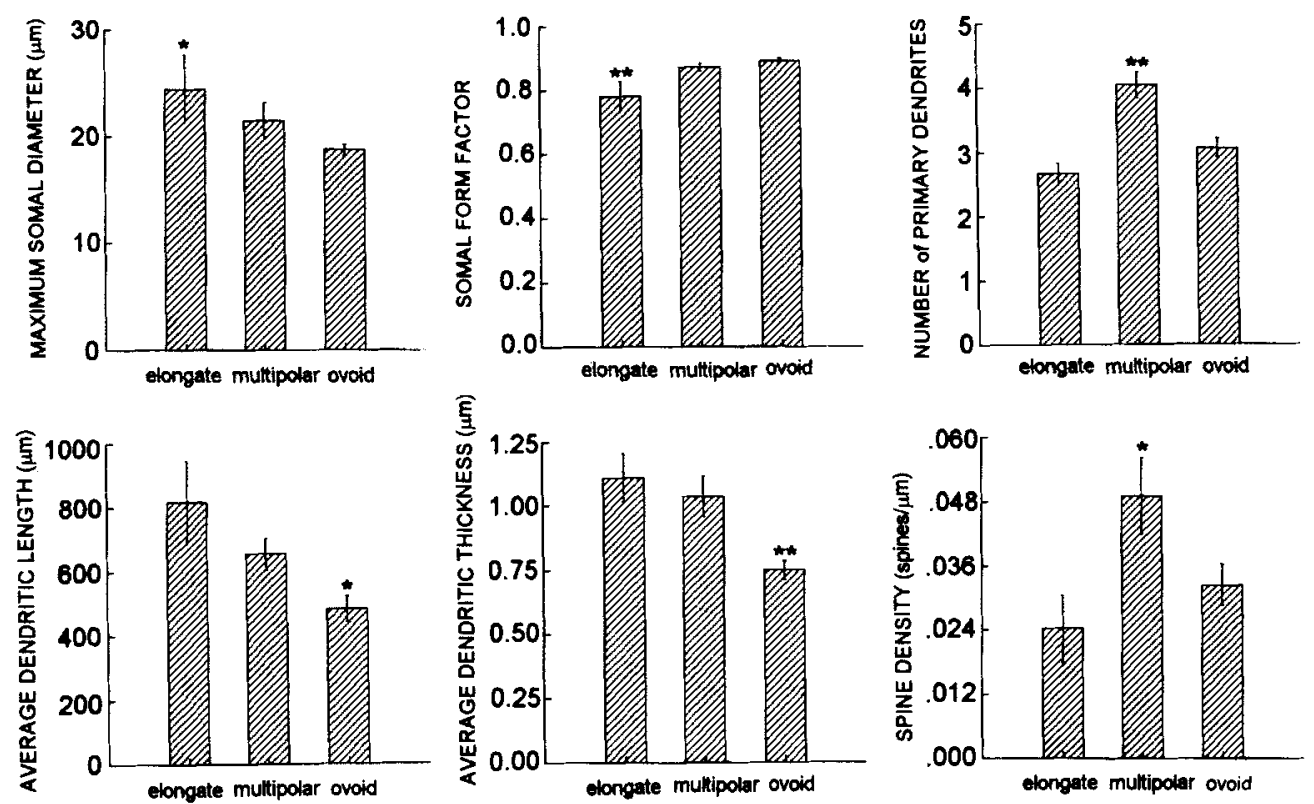

Fig. 8. Morphological differences among elongate, multipolar, and ovoid neurons. Bar graphs of six morphological parameters which were different among groups. One asterisk indicates a significant difference between that group and one other group and two asterisks indicate a significant difference from the other two groups.

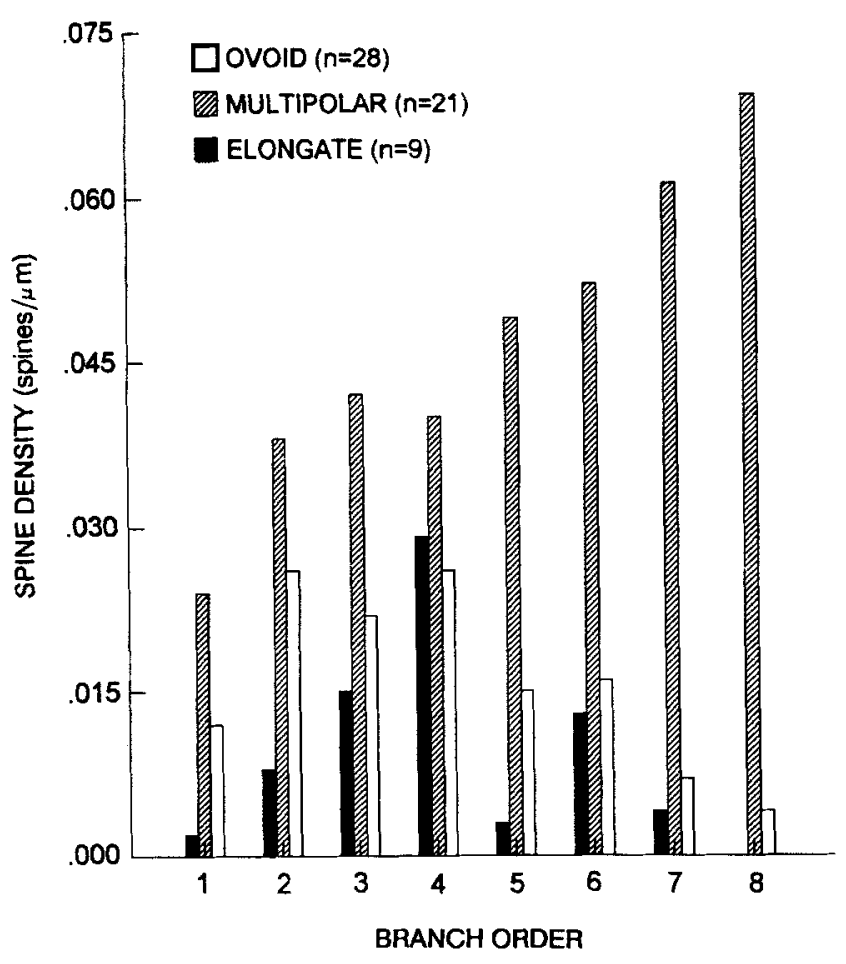

Fig. 9. Graph of the spine density versus centrifugal branch order for all elongate, multipolar, and ovoid neurons.

ovoids, DF $=3, P<.01$; Fig. 14A). Elongate neurons had either group II (44\%, Fig. 11) or III (56\%) firing patterns. The majority of multipolar neurons had a group III response (47\%, Fig. 12), but other multipolar neurons exhib- ited the firing characteristics of groups I (26\%), II (16\%), and IV $(11 \%)$. Ovoid neurons primarily had either group III $(42 \%)$ or IV $(31 \%$, Fig. 13) firing patterns and only a few of them were in groups I ( $8 \%$ ) or II (19\%).

Another biophysical property which was examined to potentially distinguish differences among the morphological groups was their responses to neurotransmitters. Substance $\mathrm{P}$ and NMDA have been previously identified as excitatory neurotransmitters in the rNTS (Wang et al., 1992; King et al., 1993) whereas GABA was inhibitory (Wang and Bradley, 1993). A high percentage ( $63 \%$ to $100 \%$ ) of each morphological type of neuron responded to these neurotransmitters (Fig. 14B, see legend for $n$ values). These results indicate that different morphological types of neurons do not respond differently to the applied neurotransmitters.

\section{DISCUSSION}

The current study is the first detailed investigation of the relationship between the structure and function of rNTS neurons. The main conclusion is that rNTS neurons with similar morphology do not have unique biophysical characteristics. Instead, each morphological group can be divided into subpopulations, each of which has different intrinsic firing properties. These subpopulations may define functional groups of neurons in the rNTS.

Numerous studies attempting to determine a relationship between the structure and function of neurons have been conducted within the last 10 years. Correlations have been found in several CNS regions, primarily within structures where the cell types are morphologically distinct, such as the cortex (Connors and Gutnick, 1990; Mason and Larkman, 1990), hippocampus (Kawaguchi and Hama, 1987; Lacaille et al., 1987; Scharfman, 1993), basolateral amygdala (Washburn and Moises, 1992; Rainnie et al., 1993), dorsal cochlear nucleus (Zhang and Oertel, 1993), 
A.
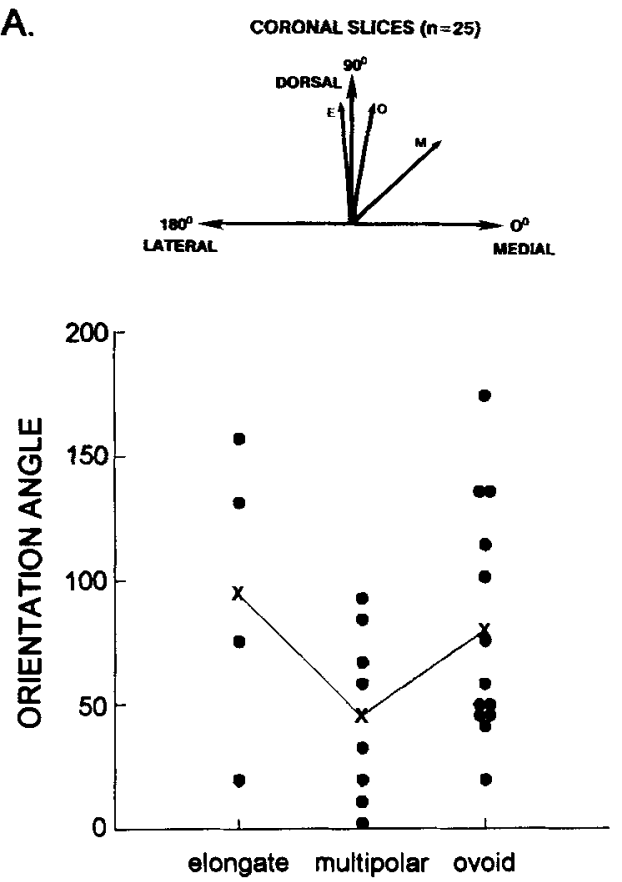

Fig. 10. Illustration of the orientation of elongate (E), multipolar $(\mathrm{M})$, and ovoid $(\mathrm{O})$ neurons in $(\mathrm{A})$ coronal and $(\mathrm{B})$ horizontal slices. The orientation angle indicates the average direction in which a neuron's dendritic field extends. In the graphs, the orientation angle of indi-
B.
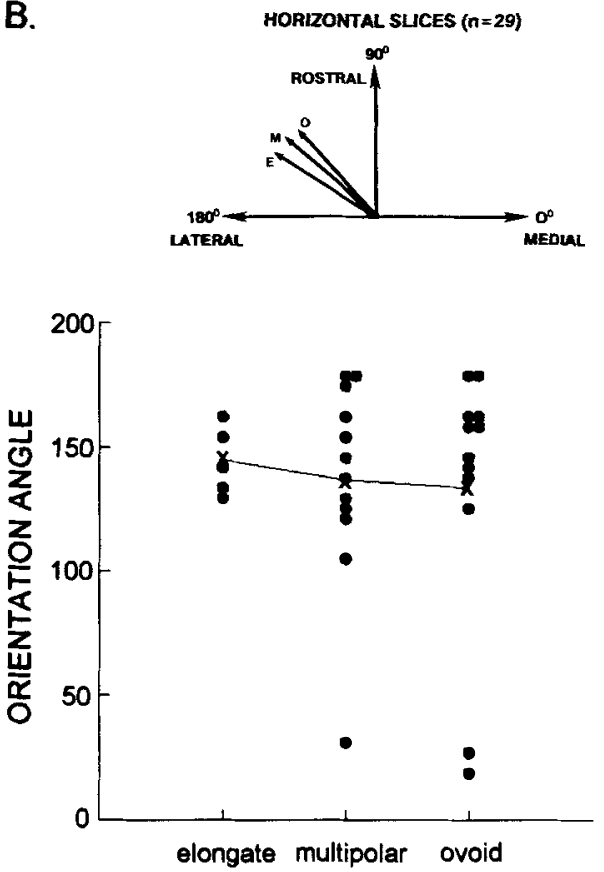

vidual neurons is represented by the solid circles and the mean orientation angle for each type of neuron is indicated by the X's. In both coronal and horizontal slices, $0^{\circ}$ indicates a medial-lateral dendritic field.
A.

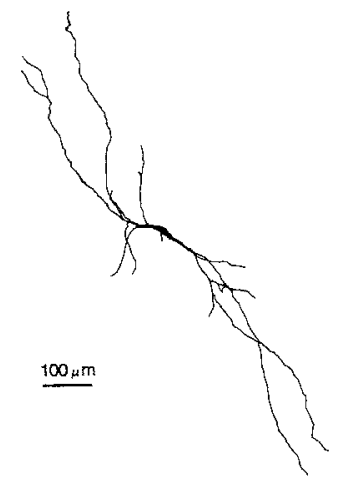

D.
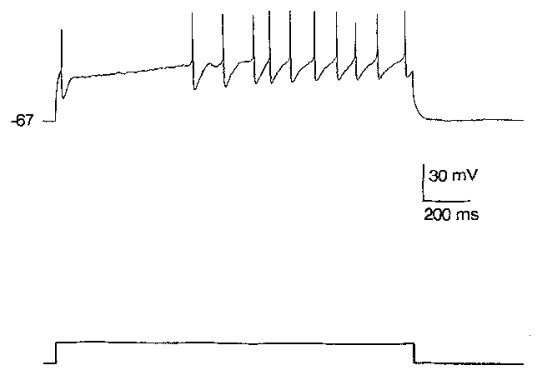

B.

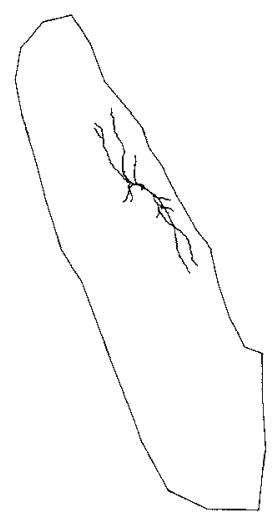

E.
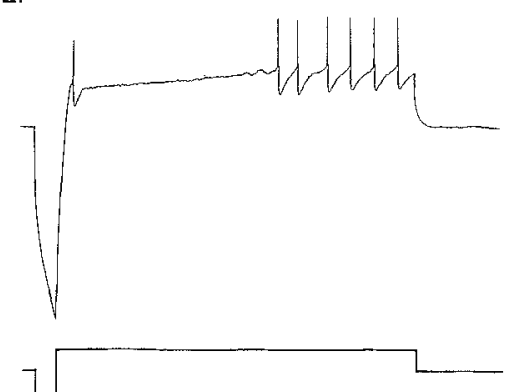

C.

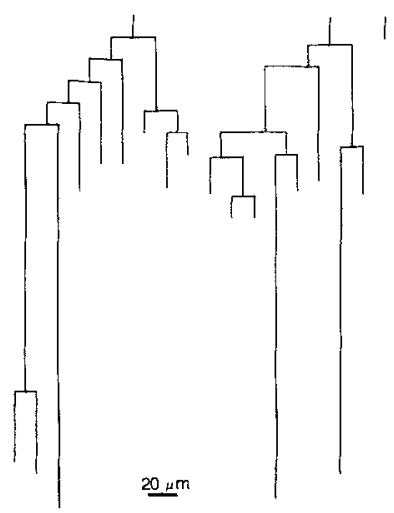

F.

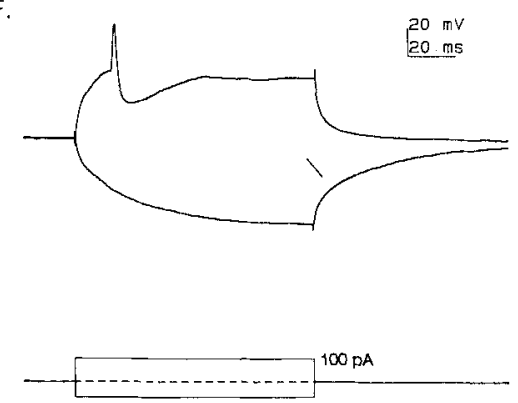

Fig. 11. Morphological and biophysical properties of an elongate neuron. A: Reconstruction of the neuron. B: Location of the neuron in a horizontal slice of the NTS. The whole left side of the nucleus is outlined. Rostral is up and medial is to the right. C: A dendrogram representation of the neuron's dendritic trees. D: The response of this neuron to a $100 \mathrm{pA}, 1,200 \mathrm{~ms}$ depolarizing current pulse. E: The response of the neuron to the depolarizing current pulse preceded by a $-200 \mathrm{pA}, 100 \mathrm{~ms}$ hyperpolarizing current pulse. Notice that this elongate neuron had the firing characteristics of group II. F: The response of this neuron to a shorter $(100 \mathrm{~ms})$ hyperpolarizing $(-50 \mathrm{pA})$ and depolarizing $(100 \mathrm{pA})$ current pulse. 
A.

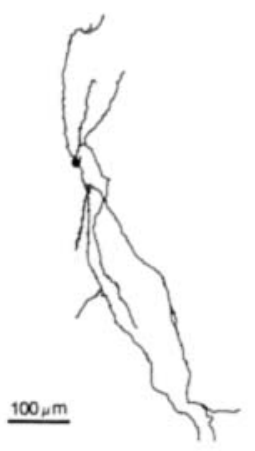

D.

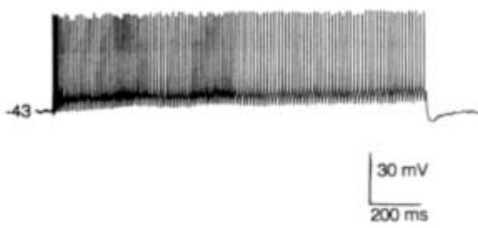

B.

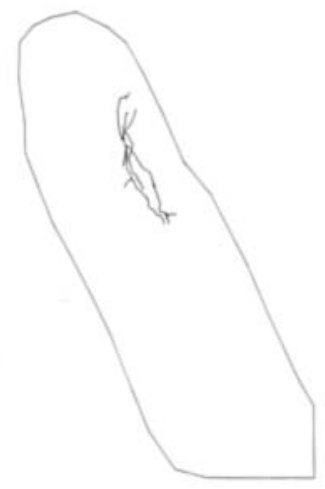

E.

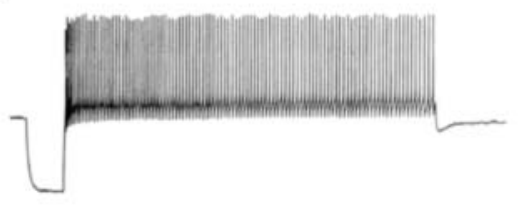

C.

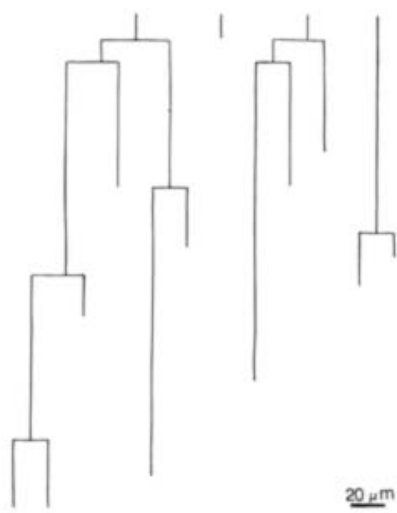

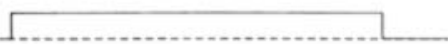
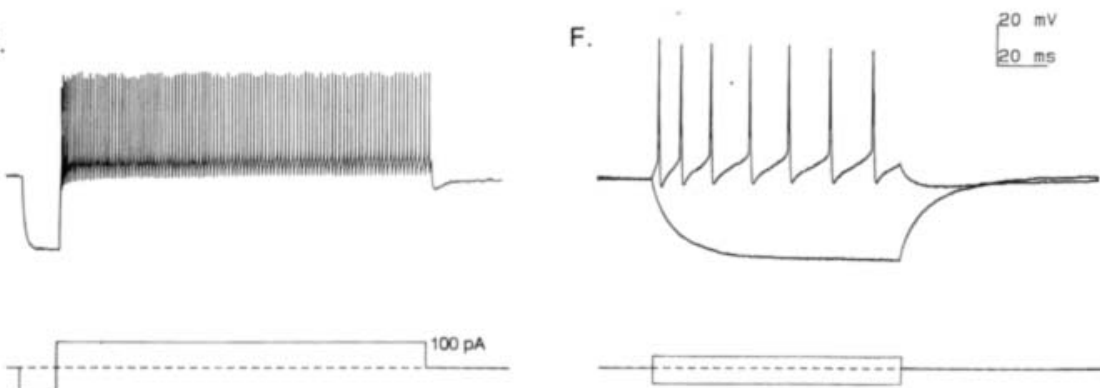

Fig. 12. Morphological and biophysical properties of a multipolar neuron. A: Reconstruction of the neuron. A-D are as in Figure 11. E: The response of the neuron to the depolarizing current pulse preceded by a $-200 \mathrm{pA}, 150 \mathrm{~ms}$ hyperpolarizing current pulse. Notice that this

multipolar neuron had the firing characteristics of group III. F: The response of this neuron to a shorter ( $100 \mathrm{~ms})$ hyperpolarizing $(-75 \mathrm{pA})$ and depolarizing ( $25 \mathrm{pA})$ current pulse.
A.

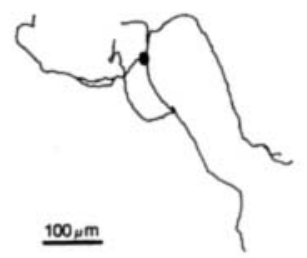

D.
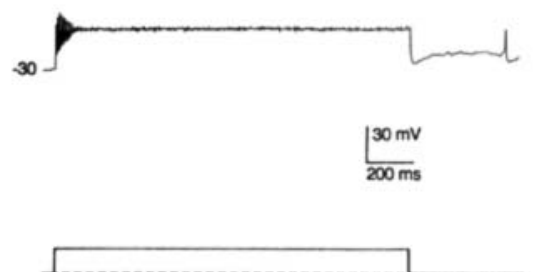

B.

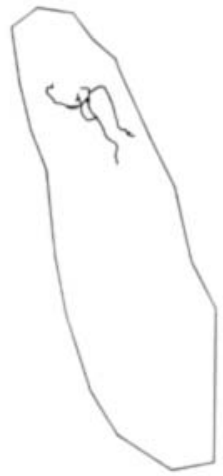

E.
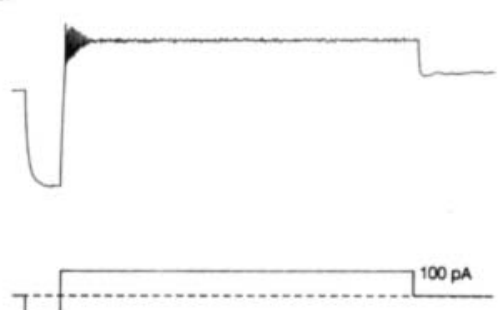

c.

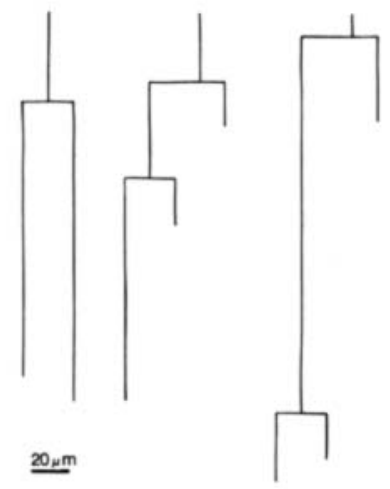

Fig. 13. Morphological and biophysical properties of an ovoid neuron. A-D are as in Figure 11. E: The response of the neuron to the depolarizing current pulse preceded by a $-200 \mathrm{pA}, 150 \mathrm{~ms}$ hyperpolarizing current pulse. Notice that this ovoid neuron had the firing characteristics of group IV. F: The response of this neuron to a shorter (100 ms) hyperpolarizing ( $-50 \mathrm{pA})$ and depolarizing ( $25 \mathrm{pA})$ current pulse. 
and olfactory bulb (Bufier et al., 1992). In contrast, a relationship between the morphology and physiology of neurons in nonlaminated structures with variable neuronal morphologies has been more difficult to define. However, correlations between structure and glucose responsiveness of neurons in the ventromedial hypothalamus (Minami et al., 1986), sensory input of spinal trigeminal neurons (Jacquin et al., 1989a,b), as well as biophysical properties and responses of neurons in the caudal (non-gustatory) NTS (Mizuno and Oomura, 1984; Champagnat et al., 1986; Dekin et al., 1987) have been reported. In the respiratory part of the caudal NTS of guinea pig, Dekin et al. (1987) proposed a strict correlation between the morphology and physiology of neurons, in which each morphological type of neuron had a unique repetitive firing pattern. However, this conclusion was based on few labeled neurons and the morphological data only consisted of a deseription of soma shape and size. Therefore, this study did not include a sufficiently rigorous morphometric analysis necessary to demonstrate differences among morphological groups. Mizuno and Oomura (1984) found a consistent difference between the morphology of caudal NTS neurons that responded to glucose and those that did not. However, they did not investigate the intrinsic biophysical properties of these neurons. Finally, Champagnat et al. (1986) reported

TABLE 2. Biophysical Properties of Elongate, Multipolar, and Ovoid Neurons ${ }^{1}$

\begin{tabular}{lccc}
\hline $\mathbf{n}$ & $\begin{array}{c}\text { Elongate } \\
(\mathbf{n}=8)\end{array}$ & $\begin{array}{c}\text { Multipolar } \\
(\mathbf{n}=\mathbf{1 5})\end{array}$ & \multicolumn{1}{c}{$\begin{array}{c}\text { Ovoid } \\
(\mathbf{n}=2)\end{array}$} \\
\hline Resting membrane potential $(\mathrm{mV})$ & $-55.8 \pm 3.6$ & $-59.2 \pm 2.3$ & $-56.5 \pm 2.4$ \\
Input resistance $(\mathrm{M} \Omega)$ & $411 \pm 59$ & $370 \pm 57^{\circ}$ & $613 \pm 72^{\mathrm{m}}$ \\
Action potential amplitude $(\mathrm{mV})$ & $\mathbf{6 8 . 0 \pm 5 . 1}$ & $71.8 \pm 3.4$ & $70.9 \pm 2.6$ \\
Action potential half-width $(\mathrm{ms})$ & $2.1 \pm 0.4$ & $1.8 \pm 0.3$ & $1.9 \pm 0.3$ \\
Action potential rise time $(\mathrm{ms})$ & $8.4 \pm 2.9$ & $14.0 \pm 4.7$ & $\mathbf{1 1 . 2} \pm 3.7$ \\
Action potential latency $(\mathrm{ms})$ & $11.4 \pm 3.6$ & $17.6 \pm 5.4$ & $\mathbf{1 4 . 0} \pm 4.0$ \\
Time constant (ms) & $14.6 \pm 2.8$ & $11.5 \pm 1.8$ & $16.6 \pm 2.0$ \\
\hline
\end{tabular}

${ }^{1}$ Data are mean $s \pm$ sem. Superscript letter 8 indicate a significant difference between the multipolar (m) and ovoid (o) groups.

A.

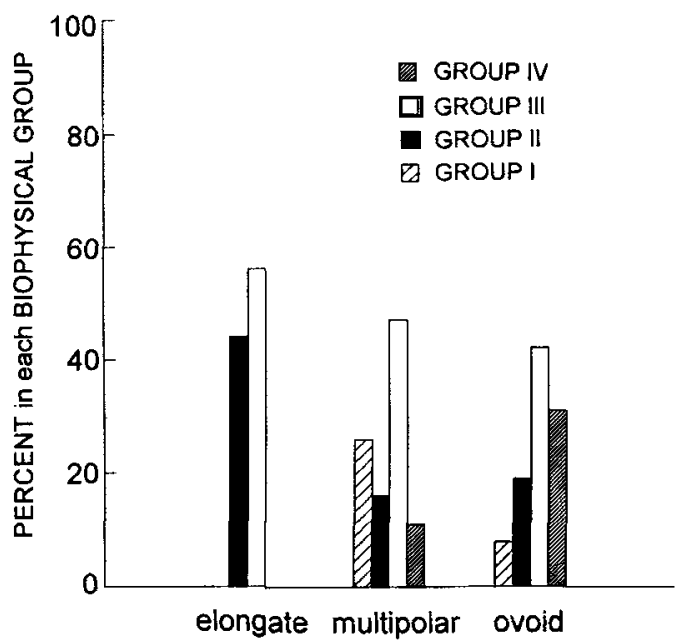

Fig. 14. A: Graph of the proportion of elongate, multipolar, and ovoid neurons having the repetitive firing characteristics of the four biophysical groups. A chi-square analysis revealed that the differences in the distributions of the three groups were significant $\left(\chi^{2}=102\right.$ for elongates, 43 for multipolars, and 24 for ovoids, $D F=3, P<.001$ ). $\mathbf{B}$. Graph of the percent of elongate, multipolar, and ovoid neurons that caudal NTS neurons with medium-sized somata and long dendrites showed prolonged reduced excitability following stimulation of the solitary tract, while neurons with small somata and short dendrites showed prolonged increased excitability. Preliminary reports from our laboratory have concluded that this probably is not the case for the rNTS, where the synaptic responses of different morphological groups of neurons, elicited by stimulation of the solitary tract, were similar (Wang et al., 1993).

\section{Classification of neuron types}

It is possible that identification of a relationship between morphology and physiology may be hindered by inadequate classification of neuron groups. We attempted to reveal groupings in the data by examining the distributions of various measurements and by performing a cluster analysis, but both of these procedures failed to identify distinct neuron groups. Therefore, we used previously established criteria for classifying both morphological (Lasiter and Kachele, 1988; Whitehead, 1988; King and Hill, 1993) and biophysical (Bradley and Sweazey, 1992) groups of neurons in the rNTS.

Elongate, multipolar, and ovoid neurons were identified by somal shape, the position from which dendrites originated from the soma, and dendritic number, thickness, and branching. The current findings are similar to earlier reports of the proportion of the three morphological groups in the rNTS (Lasiter and Kachele, 1988; Whitehead, 1988; Davis, 1993; King and Hill, 1993), indicating that our classifications were consistent with those previously reported. However, although some neurons had prototypic elongate, multipolar, and ovoid characteristics, others were more difficult to classify. They frequently did not have obvious elongate, multipolar, or ovoid morphologies, and instead presented subtle variations from one cell to the next. Fox and Powley (1992) came to a similar conclusion for the rat dorsal motor nucleus of the vagus and proposed
B.

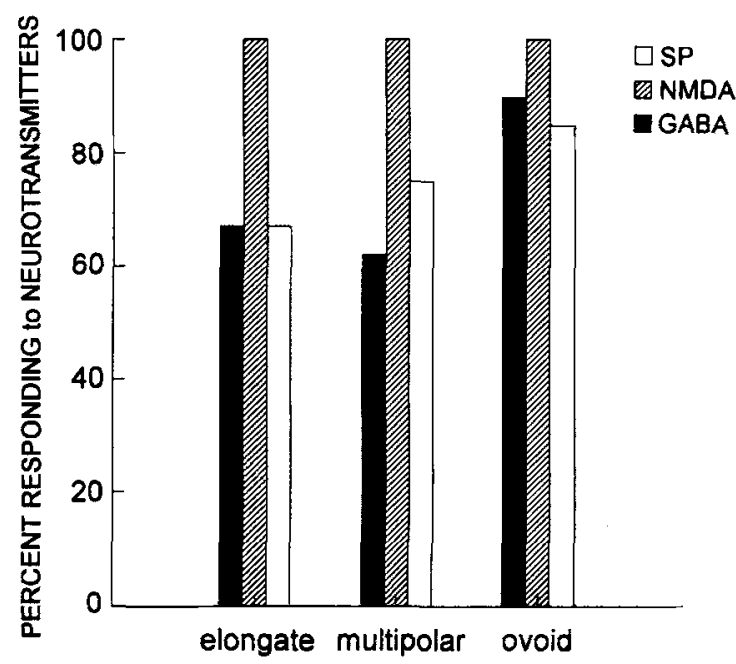

responding to substance $\mathrm{P}$ (SP), $N$-methyl-D-aspartate (NMDA), and $\gamma$-aminobutyric acid (GABA). These distributions were not statistically different. The number of elongate neurons tested with these transmitters was 3,2 , and 3 , respectively. The number of multipolar neurons tested was 4,2 , and 7 . The number of ovoid neurons tested was 11,2 , and 13. 
that the neurons do not belong to specific groups, but instead form a continuum, each cell representing a variation of a prototypic neuronal morphology. This is probably not the case in the rNTS because most neurons can be classified unequivocally and similar morphometric findings have been reported in rat (Lasiter and Kachele, 1988; King and Hill, 1993), hamster (Davis, 1988; Whitehead, 1988; Davis, 1993), and sheep (Mistretta et al., 1991).

Neurons were separated into four biophysical groups (I, II, III, and IV) on the basis of their repetitive firing patterns as previously described (Bradley and Sweazey, 1992). The different firing patterns are distinct; and consequently, the biophysical groups were easily identified. However, the intrinsic firing properties of neurons may not determine functional groups as it relates to neuron morphology. Therefore, we also evaluated the passive membrane properties and responses to neurotransmitters of rNTS neurons. It is possible, however, that a strict structure-function correlation was not determined either because we did not evaluate the appropriate biophysical property or because a combination of parameters defines functional groups. For example, responses of rNTS neurons to gustatory input may correlate to neuron morphology, but this possibility cannot be directly addressed using the slice preparation.

\section{Structure and function of rNTS neurons}

The general morphological properties of the rNTS neurons included in the current study were similar to those previously reported in rat (Lasiter and Kachele, 1988; King and Hill, 1993) and hamster (Davis, 1988; Davis and Jang, 1988; Whitehead, 1988). For example, rNTS neurons generally have simple, sparsely branched dendrites which end without extensive terminal arborizations. In addition, $r$ NTS neurons are relatively spineless, having fewer than 13 spines per $100 \mu \mathrm{m}$ of dendrite. Finally, overall, neurons are preferentially oriented in the horizontal plane and dendrites extend both medial-laterally and rostral-caudally, with the average angle of most neurons' dendritic trees being roughly parallel to their primary source of input, the solitary tract.

On the other hand, several morphometric measurements in the current investigation were different from those in previous studies. A mean total dendritic length of $1,920 \mu \mathrm{m}$ measured in biocytin-filled rNTS neurons is considerably longer than previously reported lengths in Golgi-stained preparations of the rNTS (Davis and Jang, 1988; Lasiter, 1991; King and Hill, 1993). This may be the result of more complete filling of distal dendrites with biocytin and shrinkage of the tissue caused by extensive processing during Golgi impregnation, and indicates the usefulness of biocytin as a neuronal label.

In addition, in the current study, we found several, previously unreported, anatomical differences among elongate, multipolar, and ovoid neurons (Table 1). The most striking of these is that the multipolar neurons had the greatest spine density and the elongate neurons were relatively aspiny. The distribution of spines along the dendrites of elongate, multipolar, and ovoid neurons was also different. The spine density of the most distal processes of multipolar neurons was greatest, whereas the spines on elongate and ovoid neurons were concentrated more proximally (Fig. 9). These observations suggest that multipolar neurons receive more synaptic input than the other two types and that this input is concentrated over the distal portions of their dendrites.
The biophysical characteristics of the rNTS neurons recorded in the current study were similar to those in our previous investigations in brain slices (Bradley and Sweazey, 1992; King et al., 1993; Wang and Bradley, 1993). When the biophysical properties of morphologically identified cell types were compared it was found that neurons with a particular morphology did not have unique passive membrane (Table 1) or intrinsic firing properties (Fig. 14A) nor did they respond differently to SP, NMDA, and GABA (Fig. 14B). Instead, each group contained neurons having different biophysical properties. For example, the passive membrane properties of neurons in each morphological group were variable. The only consistent difference among groups was that the ovoid neurons had the highest input resistance. In addition, neurons in each morphological group did not have identical intrinsic firing properties. Elongate neurons displayed two of the four repetitive firing patterns. Therefore, elongate neurons could be split into two subgroups, one in which the repetitive spike train was delayed by hyperpolarization (group II) and one in which it was not (group III). On the other hand, multipolar and ovoid neurons exhibited all four firing patterns. Thus, each morphological group could be divided into subpopulations, each having different intrinsic firing properties, and possibly representing functionally distinct groups. Finally, the morphological groups could not be distinguished by their responses to neurotransmitters.

Based on projection patterns, functional roles have been proposed for the cell types in the rNTS. Since elongate and multipolar neurons are retrogradely labeled following injection of tracers into the pons and brainstem (Lasiter and Kachele, 1988; Whitehead, 1990, 1993), they have been classified as the principal or projection neurons within the nucleus. On the other hand, because the ovoid neurons are not labeled following these injections they have been classified as interneurons. However, projection patterns only indicate where neurons send their output and does not indicate how neurons process sensory information. Thus, it is not surprising that principal cells and interneurons have biophysical properties unrelated to their morphology. For example, the principal neurons that project to the pons and to brainstem motor nuclei may have different biophysical properties even though they have a similar morphology. In addition, interneurons that contain different neurotransmitters or are part of different local circuits may have different firing properties. For example, $18 \%$ of the ovoid neurons in the rNTS stain positively for GABA (Lasiter and Kachele, 1988; Davis, 1993) suggesting that subpopulations of interneurons exist in the rNTS. This suggestion is supported by the current study in which the ovoid neurons had the firing patterns of all four biophysical groups. The lack of a relationship between structure and repetitive firing pattern leads to the conclusion that subpopulations of neurons exist in the rNTS, often with similar morphologies but with potentially different functional roles.

\section{Implications for the functional organization of the rNTS}

The current investigation was designed as an initial step toward understanding the neural circuits involved in processing gustatory information within the rat brainstem. These and other studies have led to the development of a working hypothesis of the neural circuitry within the rNTS (Bradley, 1993). The current analysis may have important implications for the functional organization of the rNTS. 
First, the long dendrites measured in the current study suggest that individual neurons could span a large region of the rNTS, and consequently, receive input from both the facial and glossopharyngeal nerves. Therefore, individual rNTS neurons may integrate input from the whole tongue. In fact, electrophysiological studies have suggested convergence of inputs from areas of the oral cavity innervated by the facial and glossopharyngeal nerves onto single rNTS neurons in rat (Halpern and Nelson, 1965; Travers et al., 1986) and hamster (Sweazey and Smith, 1987). In addition, the predominantly rostrocaudally extending dendrites imply that portions of the rostral and causal NTS may be interconnected. Such a connection would provide a means of integrating gustatory with general visceral information, as suggested in hamster (Beckman and Whitehead, 1991). Furthermore, this finding indicates that individual cells may extend processes into several subdivisions of the rNTS. Therefore, although the rostral central subdivision is the primary termination site of gustatory nerves (Whitehead, 1988), neurons with cell bodies in other subdivisions may receive this input on more distal processes.

Second, groups of neurons having similar morphological characteristics have different biophysical properties. Therefore, the rNTS is not simply organized into three cell types which subserve different functions. Instead, the rNTS has a very complex organization with each morphological type being divided into several subpopulations with potentially different functions.

Finally, the current investigation led to the following hypothesis about the intrinsic firing properties and synaptic interactions of proposed principal (multipolar and elongate) neurons and interneurons (ovoid) in the rNTS. The majority of principal neurons respond to depolarization with a repetitive firing pattern, which is unaltered by prior hyperpolarization. Since principal neurons in the rNTS receive direct synaptic input from primary afferent fibers (Ogawa et al., 1984; Whitehead, 1986, 1993; Wang et al., 1993; Whitehead et al., 1993), these neurons would function as a sensory relay, summing the effects of afferent inputs and transmitting afferent gustatory input out of the nucleus. Interneurons would primarily respond to excitatory afferent input with either a repetitive spike train or a short burst of action potentials. The output of these interneurons would synapse upon nearby principal neurons and potentially alter their firing patterns. Since a proportion of ovoid neurons are GABAergic (Lasiter and Kachele, 1988; Davis, 1993), they probably have an inhibitory influence on $43 \%$ of the principal neurons whose repetitive discharge pattern is modulated by membrane hyperpolarization. These changes in the firing patterns of principal neurons, caused by inhibitory inputs, would effect the output of rNTS, and therefore, alter the transmission of gustatory information to limbic, hypothalamic, and cortical structures.

\section{ACKNOWLEDGMENTS}

This work was supported by National Institutes of Health grant DC00288 to RMB.

\section{LITERATURE CITED}

Beckman, M.E., and M.C. Whitehead (1991) Intramedullary connections of the rostral nucleus of the solitary tract in the hamster. Brain Res. $557: 265-279$.
Berger, T., W. Walz, J. Schnitzer, and H. Kettenmann (1992) GABA- and glutamate-activated currents in glial cells of the mouse corpus callosum slice. J. Neurosci. Res. 4:1271-1284.

Blanton, M.G., J.J. Lo Turco, and A.R. Kriegstein (1989) Whole cell recording from neurons in slices of reptilian and mammalian cerebral cortex. I. Neurosci. Methods 30:203-210.

Bradley, R.M. (1994) Neurobiology of the gustatory zone of the nucleus tractus solitarius. In R.A. Barraco (ed): Nucleus of the Solitary Tract. Boca Raton: CRC Press, pp. 51-62.

Bradley, R.M., and R.D. Sweazey (1990) In vitro intracellular recordings from gustatory neurons in the rat solitary nucleus. Brain Res. 508:168171.

Bradley, R.M., and R.D. Sweazey (1992) Separation of neuron types in the gustatory zone of the nucleus tractus solitarii based on intrinsic firing properties. J. Neurophysiol. 67:1659-1668.

Bradley, R.M., H.M. Stedman, and C.M. Mistretta (1983) Superior laryngeal nerve response patterns to chemical stimulation of sheep epiglottis. Brain Res. 276:81-93.

Bradley, R.M., C.M. Mistretta, C.A. Bates, and H.P. Killackey (1985) Transganglionic transport of HRP from the circumvallate papilla of the rat. Brain Res. 361:154-161.

Bufler, J., F. Zufall, C. Franke, and H. Hatt (1992) Patch-clamp recordings of spiking and nonspiking interneurons from rabbit olfactory bulb slices: membrane properties and ionic currents. J. Comp. Physiol.[A] 170:145152.

Champagnat, J., M. Denavit-Saubie, K. Grant, and K.F. Shen (1986) Organiztion of synaptic transmission in the mammalian solitary complex, studied in vitro. J. Physiol. (Lond.) 381:551-573.

Connors, B.W., and M.J. Gutnick (1990) Intrinsic firing patterns of diverse neocortical neurons. Trends Neurosci. 13:99-104.

Davis, B.J. (1988) Computer-generated rotation analyses reveal a key three-dimensional feature of the nucleus of the solitary tract. Brain Res. Bull. 20:545-548.

Davis, B.J. (1993) GABA-like immunoreactivity in the gustatory zone of the nucleus of the solitary tract in the hamster: light and electron microscopic studies. Brain Res. Bull. 30:69-77.

Davis, B.J., and T. Jang (1988) A Golgi analysis of the gustatory zone of the nucleus of the solitary tract in the adult hamster. J. Comp. Neurol. 278:388-396.

Dekin, M.S. P.A. Getting, and S.M. Johnson (1987) In vitro characterization of neurons in the ventral part of the nucleus tractus solitarius. I. Identification of neuronal types and repetitive firing properties. J. Neurophysiol. 58:195-214.

Fox, E.A., and T.L. Powley (1992) Morphology of identified preganglionic neurons in the dorsal motor nucleus of the vagus. J. Comp. Neurol. 322:79-98.

Halpern, B.P., and L.M. Nelson (1965) Bulbar gustatory responses to anterior and to posterior tongue stimulation in the rat. Am. J. Physiol. 209:105-110.

Hamilton, R.B., and R. Norgren (1984) Central projections of gustatory nerves in the rat. J. Comp. Neurol. 222:560-577.

Horikawa, K., and W.E. Armstrong (1988) A versatile means of intracellular labeling: injection of biocytin and its detection with avidin conjugates. $J$. Neurosci. 25:1-11.

Housley, G.D., R.L. Martin-Body, N.J. Dawson, and J.D. Sinclair (1987) Brain stem projections of the glossopharyngeal nerve and its carotid sinus branch in the rat. Neuroscience 22:237-250.

Jacquin, M.F., J. Golden, and R.W. Rhoades (1989a) Structure-function relationships in rat brainstem subnucleus interpolaris: III. Local circuit neurons. J. Comp. Neurol. 282:24-44.

Jacquin, M.F., M. Barcia, and R.W. Rhoades (1989b) Structure-function relationships in rat brainstem subnucleus interpolaris: IV. Projection neurons. J. Comp. Neurol. 282:45-62.

Kawaguchi, Y., and K. Hama (1987) Two subtypes of non-pyramidal cells in rat hippocampal formation identified by intracellular recording and HRP injection. Brain Res. 411:190-195.

King, M.S., and R.M. Bradley (1993a) Morphology and physiology of neurons in the gustatory zone of the nucleus tractus solitarius. Chem. Senses 18:579-580.

King, M.S., and R.M. Bradley (1993b) Biophysical properties of morphologically characterized neurons of the rostral nucleus tractus solitarius (rNTS). Soc. Neurosci. Abstr. 19:1430.

King, C.T., and D.L. Hill (1993) Neuroanatomical alterations in the rat nucleus of the solitary tract following early maternal $\mathrm{NaCl}$ deprivation and subsequent $\mathrm{NaCl}$ repletion. J. Comp. Neurol. 333:531-542. 
King, M.S., L. Wang, and R.M. Bradley (1993) Substance P excites neurons in the gustatory zone of the rat nucleus tractus solitarius. Brain Res. 619:120-130.

Lacaille, J.C., A.L. Mueller, D.D. Kunkel, and P.A. Schwartzkroin (1987) Local circuit interaction between oriens/alveus interneurons and CA1 pyramidal cells in hippocampal slices: electrophysiology and morphology. J. Neurosci. 7:1979-1993.

Lasiter, P.S. (1991) Effects of early postnatal receptor damage on dendritic development in gustatory recipient zones of the rostral nucleus of the solitary tract. Dev. Brain Res. 61:197-206.

Lasiter, P.S., and D.L. Kachele (1988) Organization of GABA and GABAtransaminase containing neurons in the gustatory zone of the nucleus of the solitary tract. Brain Res. Bull. 21:623-636.

Mason, A., and A. Larkman (1990) Correlations between morphology and electrophysiology of pyramidal neurons in slices of rat visual cortex. II. Electrophysiology. J. Neurosci. 10:1415-1428.

Minami, T., Y. Oomura, and M. Sugimori (1986) Electrophysiologica properties and glucose responsiveness of guinea-pig ventromedial hypothalamic neurones in vitro. J. Physiol. (Lond.) 380:127-143.

Mistretta, C.M., S. Labyak, and M. Womble (1991) Spines are lost from neurons in nucleus of the solitary tract during a developmental period of functional convergence and complex afferent input. Chem. Senses 16:558-559.

Mizuno, Y., and Y. Oomura (1984) Glucose responding neurons in the nucleus tractus solitarius of the rat: in vitro study. Brain Res. 307:109 116.

Norgren, R. (1978) Projections from the nucleus of the solitary tract in the rat. Neuroscience 3:207-218.

Ogawa, H., T. Imoto, and T. Hayama (1984) Responsiveness of solitarioparabrachial relay neurons to taste and mechanical stimulation applied to the oral cavity in rats. Exp. Brain Res. 54:349-358.

Rainnie, D.G., E.K. Asprodini, and P. Shinnick-Gallagher (1993) Intracellular recordings from morphologically identified neurons of the basolateral amygdala. J. Neurophysiol. 69:1350-1362.

Scharfman, H.E. (1993) Spiny neurons of area CA3e in rat hippocampa slices have similar electrophysiological characteristics and synaptic responses despite morphological variation. Hippocampus 3:9-28.

Smith, D.V., and T. Hanamori (1991) Organization of gustatory sensitivities in hamster superior laryngeal nerve fibers. J. Neurophysiol. 65:10981114.

Staley, K.J., and I. Mody (1992) Shunting of excitatory input to dentate gyrus granule cells by a depolarizing $\mathrm{GABA}_{\mathrm{A}}$ receptor-mediated postsynaptic conductance. J. Neurophysiol, 68:197-212.
Sweazey, R.D., and D.V. Smith (1987) Convergence onto hamster medullary taste neurons. Brain Res, 408:173-184.

Torvik, A. (1956) Afferent connections to the sensory trigeminal nuclei, the nucleus of the solitary tract and adjacent structures. J. Comp. Neurol. 106:51-132.

Travers, J.B. (1988) Efferent projections from the anterior nucleus of the solitary tract of the hamster. Brain Res. 457:1-11.

Travers, S.P., C. Pfaffmann, and R. Norgren (1986) Convergence of lingual and palatal gustatory neural activity in the nucleus of the solitary tract. Brain Res. 365:305-320.

Wang, L., and R.M. Bradley (1993) Influence of GABA on neurons of the gustatory zone of the rat nucleus of the solitary tract. Brain Res. 616:144-153.

Wang, L., M.S. King, and R.M. Bradley (1992) Effects of excitatory and inhibitory neurotransmitters on whole cell recordings in gustatory zone of NTS. Neurosci. Abst. 18:981.

Wang, L., M.S. King, and R.M. Bradley (1993) In vitro patch clamp analysis of postsynaptic potentials mediated by excitatory and inhibitory amino acids on neurons in the gustatory zone of the nucleus tractus solitarius. Chem. Senses 18:647.

Washburn, M.S., and H.C. Moises (1992) Electrophysiological and morphological properties of rat basolateral amygdaloid neurons in vitro. J. Neurosci. 12:4066-4079.

Whitehead, M.C. (1986) Anatomy of the gustatory system in the hamster: synaptology of facial afferent terminals in the solitary nucleus. J. Comp. Neurol. 244:72-85.

Whitehead, M.C. (1988) Neuronal architecture of the nucleus of the solitary tract in the hamster. J. Comp. Neurol. 276:547-572.

Whitehead, M.C. (1990) Subdivisions and neuron types of the nucleus of the solitary tract that project to the parabrachial nucleus in the hamster. J. Comp. Neurol. 301:554-574.

Whitehead, M.C. (1993) Distribution of synapses on identified cell types in a gustatory subdivision of the nucleus of the solitary tract. J. Comp. Neurol. 332:326-340.

Whitehead, M.C., and M.E. Frank (1983) Anatomy of the gustatory system in the hamster: central projections of the chorda tympani and lingual nerve. J. Comp. Neurol. 220:378-395.

Whitehead, M.C., M. McPheeters, L.D. Savoy, and M.E. Frank (1993) Morphological types of neurons located at taste-responsive sites in the solitary nucleus of the hamster. Microsc. Res. Tech, 26:245-259.

Zhang, S., and D. Oertel (1993) Cartwheel and stellate cells of the dorsal cochlear nucleus of mice: intracellular recordings in slices. J. Neurophysiol. 69:1384-1397. 